\title{
The Effects of Superior Temporal Cortex Lesions on the Processing and Retention of Auditory Information in Monkeys (Cebus apella)
}

\author{
Michael Colombo, ${ }^{1}$ Hillary R. Rodman, ${ }^{2}$ and Charles G. Gross ${ }^{2}$ \\ ${ }^{1}$ Department of Psychology, Rutgers University, New Brunswick, New Jersey 08903, and 2Department of Psychology, \\ Princeton University, Princeton, New Jersey 08544
}

\begin{abstract}
Three monkeys with extensive preoperative training on visual and auditory memory tasks (delayed matching-to-sample), an auditory pattern-discrimination task, and a visual serial-order task, received bilateral lesions of the superior temporal (ST) cortex in two stages, with testing after each lesion. Unilateral ST cortex lesions resulted in only moderate auditory memory impairments, whereas bilateral ST cortex lesions resulted in severe auditory memory impairments. The bilateral ST cortex lesions also resulted in severe impairments in the ability to relearn the auditory pattern-discrimination task. In contrast to
\end{abstract}

the auditory impairments, neither unilateral nor bilateral ST cortex lesions had any effect whatsoever on either visual memory or visual serial-order behavior. These findings indicate that the ST cortex plays a role in auditory processing and retention similar to that played by the inferior temporal cortex for visual processing and retention.

Key words: auditory cortex; auditory memory; visual memory; delayed matching-to-sample; serial-order task; pattern discrimination; frequency discrimination
Much of what we know regarding the neural basis of information processing in the primate brain is based on studies of the visual system. For example, it is well established that damage to the inferior temporal (IT) cortex, a high-level visual area, results in impairments on both visual discrimination and visual memory tasks (Gross, 1973; Mishkin, 1982). Less well established is the role played by the superior temporal (ST) cortex, a high-level auditory area, in the processing and retention of auditory information in monkeys. In fact, despite similarities between the visual and auditory cortex in terms of their cortical projection patterns (Pandya and Kuypers, 1969; Jones and Powell, 1970; Pandya et al., 1988; Felleman and Van Essen, 1991), there is little evidence that ST cortex lesions in monkeys impair performance on auditorydiscrimination tasks, and there is no evidence that such lesions impair performance on auditory memory tasks.

The reason that ST cortex lesions have been found to have little effect on auditory behavior in monkeys may be traced to procedural problems with the auditory tasks used. Consider, for example, the findings that auditory cortex lesions have little effect on auditory discriminative behavior in monkeys. One problem may be that in most studies monkeys were trained to discriminate on the basis of differences in the frequency of two tones. Such simple frequency discriminations survive not only ST cortex lesions (Weiskrantz and Mishkin, 1958; Iversen and Mishkin, 1973; Pratt and Iversen, 1978), but in many cases they can also survive extensive damage to primary auditory cortex (Evarts, 1952; Jerison and Neff, 1953; Wegener, 1968; Dewson et al., 1969; Pratt and

Received Dec. 14, 1995; revised April 22, 1996; accepted May 3, 1996.

This research was supported by National Science Foundation Grant 8417383 to M. R. D'Amato and National Institutes of Health Grant MH-19420 to C.G.G. We thank

M. R. D'Amato for providing valuable input during all phases of this project, L.

Kuseryk for help with the histology, and E. Yeterian and D. Pandya for providing assistance with the cortical reconstructions.

Correspondence should be addressed to Michael Colombo, Department of Psychology, University of Otago, Dunedin, New Zealand.

Dr. Rodman's present address: Department of Psychology, Emory University, Atlanta, GA 30322.

Copyright (C) 1996 Society for Neuroscience $0270-6474 / 96 / 164501-17 \$ 05.00 / 0$
Iversen, 1978). By contrast, much greater deficits are observed after auditory cortex lesions when monkeys are trained to discriminate on the basis of differences in acoustic patterns rather than between differences in frequency (Jerison and Neff, 1953; Neff, 1961a,b; Dewson et al., 1969; Pratt and Iversen, 1978; Heffner and Heffner, 1984, 1986a). Unfortunately, the extent to which the ST cortex alone is necessary for auditory pattern discriminations is unclear, because in most studies the lesions either were restricted to primary auditory cortex (Pratt and Iversen, 1978) or included primary auditory cortex in addition to ST cortex (Jerison and Neff, 1953; Neff, 1961a,b).

A procedural-based explanation can also be offered for the finding that in contrast to the visual memory deficits that follow IT cortex lesions in monkeys (Mishkin, 1982), there is no evidence that auditory memory in monkeys is impaired by ST cortex lesions. Part of the problem may be that the tasks used to tap auditory memory could have been solved using visual or spatial memory rather than auditory memory. The reason is as follows. In most memory/lesion studies, memory is assessed using the delayed matching-to-sample (DMS) procedure. Although there are many variations of the DMS procedure, the basic task is quite straightforward. A sample stimulus is presented to the subject for a brief period of time and then removed. After a retention period, two comparison stimuli are presented, one the same as the sample stimulus and one different from the sample stimulus, and the subject is required to choose the comparison stimulus that matches the previously displayed sample stimulus. In the studies that have examined the effects of IT cortex lesions on visual memory, the monkeys were trained on visual DMS tasks in which the sample stimuli and the comparison stimuli were visual. In contrast, in all previous studies that examined the effects of ST cortex lesions on auditory memory, monkeys were trained on DMS procedures in which the sample stimuli were auditory but the comparison stimuli were either visual (auditory-visual DMS procedures: Dewson, 1977, 1978, 1979; Costalupes, 1984) or spatial (auditory-spatial DMS procedures: Dewson et al., 1970; 
Cowey and Dewson, 1972; Cowey and Weiskrantz, 1976; Pratt and Iversen, 1978).

It is well known that monkeys can solve DMS tasks by engaging in retrospective processing, i.e., remembering aspects of the sample stimulus during the delay period (D'Amato, 1973; Roberts and Grant, 1976), or by engaging in prospective processing, i.e., remembering aspects of the comparison stimuli during the delay period (Gaffan, 1977; Honig, 1978; Roitblat, 1980; Honig and Thompson, 1982; Colombo and Graziano, 1994). If the monkeys in the ST cortex lesion studies were engaging in retrospective processing, then the auditory-visual and auditory-spatial DMS tasks indeed would have tapped auditory memory. If the monkeys were using auditory memory, then the findings that ST cortex lesions had no effect on auditory-visual or auditory-spatial DMS memory performance would indicate correctly that the ST cortex does not play a role in auditory memory. On the other hand, if the monkeys were engaging in prospective processing, then the auditory-visual and auditory-spatial DMS tasks would have tapped visual and spatial memory, respectively, and not auditory memory. If the monkeys were bypassing auditory memory, then the findings that ST cortex lesions had little or no effect on performance of the auditory-visual and auditory-spatial DMS tasks would not be surprising, and in fact, would be expected. ${ }^{1}$

The main purpose of the present experiment was to examine whether ST cortex lesions impaired auditory memory in monkeys using a task that was known to tap auditory memory. This was achieved by training monkeys on an auditory DMS task in which the sample and comparison stimuli were both auditory. With such a task, whether the monkeys engage in retrospective or prospective processing is irrelevant, because in both cases the task would require auditory memory. A second purpose was to examine whether ST cortex lesions impaired auditory pattern discrimination in monkeys. As a control for the auditory DMS task, all of the monkeys were also trained on a procedurally identical visual DMS task. In addition, the monkeys were also trained on a visual serial-order task.

Parts of this paper have been published previously (Colombo et al., 1990).

\section{MATERIALS AND METHODS}

\section{Subjects}

Three laboratory-born New World monkeys (Cebus apella), ranging in age from 7 to 9 years at the start of the study, served as subjects. The study took $\sim 1$ year to complete. During that time, the subjects were housed individually with water available continuously and maintained on a $12 \mathrm{hr}$ light/dark cycle with lights on at $7 \mathrm{~A} . \mathrm{M}$. They received one feeding per day (Purina Monkey Chow 5045), 2-3 hr after a test session, in an amount adjusted to support reliable and stable performance. The daily rations were sufficient to maintain the monkeys at $\sim 90 \%$ of their freefeeding body weights. Their diet was supplemented with fresh fruit and raisins.

With the exception of the frequency-discrimination task, all three monkeys had been trained for a period of 5 years on all of the tasks used

\footnotetext{
${ }^{1}$ One study that seemed free of the recoding interpretational problem was that of Stepien et al. (1960), in which monkeys were trained on a DMS task to match auditory pulsed stimuli of 5 and $20 \mathrm{~Hz}$. According to the study, bilateral "superior temporal" lesions resulted in an apparent inability to perform the auditory DMS task. Unfortunately, the results are inconclusive with respect to auditory memory for several reasons. First, it is unclear whether click rate requires uniquely auditory processing, because a general mechanism may exist for encoding repetitive stimuli independent of the associated modality (Ravizza and Belmore, 1978). Consistent with this possibility is the finding that before the operation, the animals generalized immediately from auditory clicks to visual flashes of the same frequency. Second, and more importantly, a 2-year follow-up study (Cordeau and Mahut, 1964) revealed no auditory DMS deficit but rather a visual DMS impairment, a finding not surprising given that the "auditory cortex" lesion actually included the dorsal part of IT cortex and the temporal pole and in fact very little of the ST cortex
}
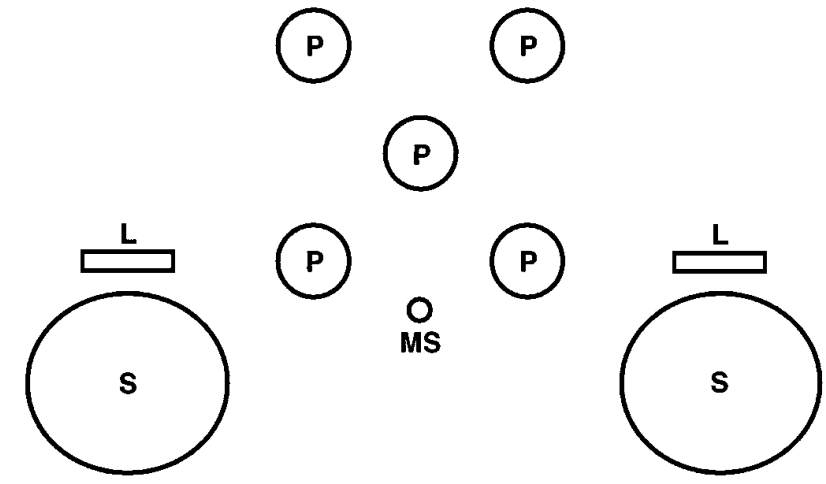

Figure 1. Diagram of the front panel of the monkey operant chamber. $P$ refers to the visual stimulus projectors used for the visual DMS task and visual serial-order task. $S$ and $L$ refer to the speakers and levers, respectively, used for the auditory DMS, pattern-discrimination, and frequencydiscrimination tasks. $M S$ refers to a microswitch that was used to initiate trials on the serial-order, pattern-discrimination, and frequencydiscrimination tasks.

in the present study. Their first experience was in an auditory study discriminating various tunes (D'Amato and Salmon, 1982, 1984). All three monkeys were then trained on visual and then auditory DMS tasks with delays ranging from 0 to $32 \mathrm{sec}$ (D'Amato and Colombo, 1985; D'Amato et al., 1985; Colombo and D'Amato, 1986). They next participated in another auditory study discriminating between a series of ascending and descending tones (D'Amato and Colombo, 1988a). Finally, before the present study, all three monkeys were trained on the visual serial-order task.

\section{Apparatus}

All testing was conducted in two Lehigh Valley monkey-operant chambers (Model 1317). The front panel (Fig. 1) of each box housed five inline visual stimulus projectors (IEE Model 1071) arranged at the midpoint and four corners of a $12 \mathrm{~cm}$ square. Each projector was fitted with a transparent plastic button that served as the response mechanism for the visual tasks. Situated $16 \mathrm{~cm}$ on either side of the two bottom projectors were levers that served as the response mechanisms for the auditory tasks. Beneath each lever was a speaker $(3 \mathrm{~W}, 10.2 \mathrm{~cm}, 8 \mathrm{ohm}$, Realistic Model FE-103) that was used to deliver the acoustic stimuli. Located directly below the projector array was a microswitch that was used to initiate the trials on the serial-order and auditory-discrimination tasks.

Each chamber was illuminated by an overhead houselight, a $25 \mathrm{~W}$ soft-white light bulb situated behind a transparent plastic shield. The houselight could be dimmed by adding a $500 \mathrm{ohm}$ resistor in series with the bulb. Extraneous sounds were masked by white noise and an exhaust fan, which together measured $75 \mathrm{~dB}$. Noyes banana-flavored pellets $(190$ $\mathrm{mg}$ ), delivered to a food cup on the lower part of the right wall, served as reinforcers. The programming of trial events, presentation of stimuli, and data recording were controlled by a PDP 8/e minicomputer (Digital Equipment Corporation) and a Commodore PET microcomputer (series 2001) and disk unit (Model 8050).

Visual stimuli. The visual stimuli consisted of colors and simple geometric forms such as a circle, a plus sign, a vertical line, a red disk, a dot, and an hourglass. All form stimuli appeared as white figures on a black background and were composed of lines $1.5 \mathrm{~mm}$ wide $\times 17 \mathrm{~mm}$ long. The circle, also composed of a $1.5 \mathrm{~mm}$ wide line, was $17 \mathrm{~mm}$ in diameter. The solid dot and red disk were $6 \mathrm{~mm}$ and $25 \mathrm{~mm}$ in diameter, respectively.

Auditory stimuli. With one exception noted below, all of the auditory stimuli consisted of square-wave tones of different frequencies. The sounds were generated by a Commodore Pet (series 2001) microcomputer and amplified by the amplifier portion of an MTU D/A converter (Model K-1002). On one brief occasion, monkey $\mathrm{T}$ was tested with sine-wave tones rather than square-wave tones. These sounds were generated by a Heath-Schlumberger (Model SG-18A) sine-wave generator and amplified by a Realistic (Model SA-10) stereo amplifier. The intensity of all the acoustic stimuli ranged from 78 to $85 \mathrm{~dB}$, as measured on the C scale (slow setting) of a Simpson sound lever meter (Model 886) placed $15 \mathrm{~cm}$ in front of the center of the right speaker and $23 \mathrm{~cm}$ above the chamber floor. 


\section{Behavioral tasks}

Auditory DMS. The main stimuli used on the auditory DMS task consisted of a high-frequency tone (HT) of $3676 \mathrm{~Hz}$ and a low-frequency tone (LT) of $243 \mathrm{~Hz}$. The monkeys were trained on a Konorski (1959) successive go/no-go asymmetrically reinforced matching procedure. The sequence of events on an auditory matching trial was as follows. At the end of a $20 \mathrm{sec}$ intertrial interval (ITI), the houselight was illuminated, and $2 \mathrm{sec}$ later a sample stimulus was played from the speaker located beneath the right lever. After a $2 \mathrm{sec}$ listening period, the first response to the right lever terminated the sample stimulus, extinguished the houselight, and initiated a delay interval. At the end of the delay, the houselight was illuminated concurrently with presentation of the comparison stimulus from the speaker situated under the left lever. After a $0.4 \mathrm{sec}$ listening period, the comparison stimulus continued to play until a response was made to the left lever, or until an additional $2.6 \mathrm{sec}$ (the response window) elapsed.

On matching trials (HT-HT and LT-LT), a correct response was defined as pressing the left lever during the response window, which resulted in termination of the comparison stimulus, delivery of reward, and entry into the ITI, signaled by dimming of the overhead houselight. On nonmatching trials (HT-LT and LT-HT), a correct response required withholding presses to the left lever during the response window. Such action resulted in termination of the comparison stimulus and entry into the ITI, but no reward pellet was delivered. Withholding a response on matching trials, or responding on nonmatching trials, both defined as incorrect responses, resulted in a $60 \mathrm{sec}$ time-out period, during which the houselight was turned off. The time-out period was followed by the ITI.

All auditory DMS sessions consisted of 39 trials, the first three of which served to familiarize the subject with the conditions of the task and therefore were not included in the data analysis. The remaining 36 trials were divided equally among the four possible sample/comparison configurations with the only restriction that no more than three matching or three nonmatching trials appear in succession.

The auditory DMS task was administered in two different formats. In the "baseline" format, the delay for all trials was set at $0.5 \mathrm{sec}$ in duration. In the "retention" format, three delay periods were used $(0.5,4$, and 32 $\mathrm{sec}$ ), with 12 trials dedicated to each delay period, intermixed quasirandomly; the only restriction was that no more than three delays of a kind or three matching or nonmatching trials appear in succession. Throughout the study, eight different pseudorandomly constructed sequences of trials were used for baseline DMS testing, and 12 different pseudorandomly constructed sequences were used for the retention DMS testing.

Visual DMS. The stimuli used on the visual DMS task consisted of a plus and a circle. Procedurally, the visual and auditory DMS tasks were identical, with the exception that the sample and comparison visual stimuli were presented on the bottom right and left projectors, respectively, and the monkeys responded to the visual stimuli by directly pressing the plastic buttons that faced the projectors.

Auditory pattern and frequency discriminations. Two different types of stimuli were used for auditory-discrimination testing. For the auditorypattern discrimination, the animals were trained to discriminate between an ascending $(\mathrm{S}+$ : $2688-3012-3378-3788 \mathrm{~Hz})$ and a descending $(\mathrm{S}-$ : 4808-4237-3788-3378 Hz) series of tones, which correspond to exemplars A1 and D3 of the D'Amato and Colombo (1988a) study. The duration of each tone was $140 \mathrm{msec}$ separated by an $80 \mathrm{msec}$ silent period for a total playing time of $800 \mathrm{msec}$. Consecutive playing times were separated by an $800 \mathrm{msec}$ silent period. For the frequency discrimination, the animals were required to discriminate between either two highfrequency notes $(\mathrm{S}+, 4808 \mathrm{~Hz} ; \mathrm{S}-, 2688 \mathrm{~Hz})$ or two low-frequency notes $(\mathrm{S}+, 613 \mathrm{~Hz} ; \mathrm{S}-, 306 \mathrm{~Hz})$. None of the animals had any experience with the frequency-discrimination task before the start of this study.

Only the right lever and the right speaker were used for the pattern and frequency-discrimination tasks. The monkeys were trained on an asymmetrically rewarded go/no-go procedure to press the lever in the presence of the $\mathrm{S}+$ sound and to refrain from pressing when the $\mathrm{S}-$ sound was presented. The sequence of events on a discrimination trial was as follows. At the end of a $15 \mathrm{sec}$ ITI, the houselight was illuminated, and after four presses to the microswitch, the auditory stimulus was played from the speaker situated beneath the right lever. The stimulus was played for both a listening period, during which responses to the lever were ineffective, and a response period, during which responses were effective. For the pattern-discrimination task, the listening and response periods were $2.5 \mathrm{sec}$ and $3.1 \mathrm{sec}$, respectively; for the frequencydiscrimination task, these values were $0.4 \mathrm{sec}$ and $2.6 \mathrm{sec}$, respectively.

$\mathrm{On} \mathrm{S}+$ trials, a correct response, defined as pressing the lever during the response period, resulted in termination of the $\mathrm{S}+$ stimulus, delivery of the reward pellet, and entry into the ITI, signaled by dimming of the overhead houselight. A correct response on an S- trial required withholding a response to the lever for the duration of the response period, which resulted in entry into the ITI but no reward. Failure to press during $\mathrm{S}+$ trials or pressing during $\mathrm{S}-$ trials, both defined as incorrect responses, resulted in a $60 \mathrm{sec}$ time-out period, during which the houselight was turned off.

Each session consisted of 48 trials, $24 \mathrm{~S}+$ and $24 \mathrm{~S}-$ trials intermixed randomly, with the only restriction being that no more than three $\mathrm{S}+$ or three $\mathrm{S}-$ trials occurred in succession. Five different pseudorandomly constructed sequences of $\mathrm{S}+$ and $\mathrm{S}-$ presentations were used for the pattern-discrimination task, and five different pseudorandomly constructed sequences were used for the frequency-discrimination task.

Visual serial order. The stimuli used on the serial-order task consisted of a vertical line, a red disk, a dot, and an hourglass, which for ease of exposition will hereafter be referred to as A, B, C, and D, respectively. A serial-order trial proceeded in the following manner. At the end of a 15 sec ITI, the houselight was illuminated, and after completion of four presses to the microswitch, all four stimuli were presented simultaneously on any four of the five projectors. A correct response required pressing the four stimuli in the order $\mathrm{A} \rightarrow \mathrm{B} \rightarrow \mathrm{C} \rightarrow \mathrm{D}$. Such correct responses resulted in termination of the stimulus display, delivery of reward, and entry into the ITI, signaled by dimming of the overhead houselight. Any deviation from the $\mathrm{A} \rightarrow \mathrm{B} \rightarrow \mathrm{C} \rightarrow \mathrm{D}$ response sequence (e.g., by committing a forward error such as $\mathrm{A} \rightarrow \mathrm{B} \rightarrow \mathrm{D}$, or a backward error such as $\mathrm{A} \rightarrow \mathrm{B} \rightarrow \mathrm{C} \rightarrow \mathrm{B}$ ) was considered an incorrect response and resulted in termination of the trial and commencement of a $60 \mathrm{sec}$ time-out period, during which the houselight was turned off. The ITI followed the time-out period. Immediately repeating a response to a stimulus (e.g., $\mathrm{A} \rightarrow \mathrm{B} \rightarrow \mathrm{B} \rightarrow \mathrm{C} \rightarrow \mathrm{D})$ was not considered an error. Each session consisted of 40 trials, with the four stimuli appearing in different spatial locations on each trial. If a stimulus array is defined as the arrangement of the four stimuli on the five projectors, then 120 different spatial arrays are possible. A subset of 40 arrays were chosen randomly from the complete set, and four different pseudorandomly constructed sequences of these 40 arrays were used throughout the experiment.

Once the monkeys learned the ABCD serial-order task, they were tested for their knowledge of serial order using a pairwise test. The procedure for the pairwise test was identical to that described for the serial-order task, with the exception that only two of four stimuli were presented on a trial; which two stimuli were presented changed from trial to trial. A correct response required that the monkeys respond to the two stimuli in the order in which they appeared in the original sequence. A pairwise test session consisted of 36 trials, with six trials dedicated to each of the six possible pairs of stimuli that can be generated from the $\mathrm{ABCD}$ series $(\mathrm{AB}, \mathrm{AC}, \mathrm{AD}, \mathrm{BC}, \mathrm{BD}$, and $\mathrm{CD})$.

\section{Experimental design}

All three animals were tested preoperatively on the following tasks in the following order: visual DMS, auditory DMS, pattern discrimination, and serial order. After establishing baseline performance on these tasks, the animals received a two-stage lesion of the ST cortex with testing after each operation. Postoperatively the animals were tested in the same order as described for preoperative testing, with the exception that testing on the frequency- and pattern-discrimination tasks occurred only after the second operation.

Visual and auditory DMS tasks. For both visual and auditory DMS tasks, the subjects were first required to satisfy a criterion of one session with at least 32 of 36 correct responses ( $88.9 \%$ correct) on the baseline DMS task in which all of the delays were set at $0.5 \mathrm{sec}$. The animals were then tested on the retention DMS task with delays of $0.5,4$, and $32 \mathrm{sec}$. The first two sessions of visual and auditory retention DMS testing served to familiarize the subject with the task and were therefore not included in the data analysis. Testing continued in replications of four sessions, with the performance during each replication averaged to yield a retention gradient. Preoperatively, testing continued until stable retention gradients were generated. After the operations, testing continued until the postoperative retention performance at each delay was within one correct response $(2.1 \%)$ of the mean preoperative retention gradient performance.

Pattern-discrimination task. After auditory DMS testing, the animals were tested on the pattern-discrimination task until they satisfied a criterion of one session with at least 43 out of 48 correct responses $(89.6 \%$ correct). 
Table 1. Percentage of auditory cortex damaged

\begin{tabular}{|c|c|c|c|c|c|c|c|c|c|}
\hline & \multicolumn{3}{|c|}{ Monkey M } & \multicolumn{3}{|c|}{ Monkey T } & \multicolumn{3}{|c|}{ Monkey B } \\
\hline & Right & Left & Total & Right & Left & Total & Right & Left & Total \\
\hline LS & 14.9 & 14.5 & 14.7 & 33.2 & 22.6 & 27.8 & 36.8 & 24.7 & 30.7 \\
\hline ST cortex & 90.0 & 90.2 & 90.1 & 85.9 & 78.7 & 82.3 & 89.0 & 87.9 & 88.5 \\
\hline STS & 89.2 & 29.3 & 58.3 & 38.8 & 39.9 & 39.3 & 40.1 & 48.2 & 44.2 \\
\hline All areas & 63.3 & 43.3 & 53.4 & 53.1 & 46.5 & 49.8 & 56.2 & 52.4 & 54.3 \\
\hline
\end{tabular}

LS, Lower bank of lateral sulcus; ST, superior temporal; STS, upper bank of superior temporal sulcus.

Serial-order task. After pattern-discrimination testing, the animals were tested on the serial-order task until they satisfied a criterion of one session with at least 32 out of 40 correct responses (80\% correct). Once this was achieved, the animals were given two pairwise tests separated by $3 \mathrm{~d}$ of testing on the ABCD serial-order task.

\section{Surgery}

Before surgery, the monkeys were treated with atropine sulfate $(0.5$ $\mathrm{ml} / \mathrm{kg}$, i.m. ), furosemide $(0.5 \mathrm{ml} / \mathrm{kg}$, i.m.), and ketamine hydrochloride ( 20 $\mathrm{mg} / \mathrm{kg}$, i.m.) and then given halothane via an endotracheal tube to maintain deep anesthesia. The monkeys were then placed in a headholder, immobilized with pancurium bromide $\left(0.02 \mathrm{mg} \cdot \mathrm{kg}^{-1} \cdot \mathrm{hr}^{-1}\right)$, respirated with $68.5 \%$ nitrous oxide and $30.0 \%$ oxygen, and anesthetized with $1.5 \%$ halothane.

All surgery was conducted under aseptic conditions. An incision was first made in the scalp exposing the dorsal surface of the skull. The temporal muscle was then retracted, and the bone overlying the ST cortex was removed with rongeurs. The dura was then cut and retracted, and the cortical tissue was removed by subpial aspiration with the aid of an operating microscope. On completion of tissue removal, the dura was sutured closed, and the temporal muscle was sewn back in place. The scalp incision was then closed in anatomical layers, and a topical analgesic (Xylocaine) was administered to the wound. On recovery of spontaneous breathing, the monkey was maintained in a heated padded cage, observed until it was alert and mobile, and then placed in its home cage for the remainder of the postoperative recovery period. Aspirin in liquid form was given immediately on recovery and continued for several days postoperatively.

Monkeys B and T received lesions of the left ST cortex first, whereas monkey M received lesions of the right ST cortex first. The lesions were intended to remove auditory "association" cortex (area TA) but to spare primary (area TC) and secondary (area TB) auditory cortex. In terms of the architectonic parcellation scheme of Sanides (1972) and Pandya and Sanides (1973), the intention was to remove areas Ts1, Ts2, Ts3, and Tpt, sparing the proisocortex of the temporal pole (Pro), auditory koniocortex (Kam and Kalt), parakoniocortex (paAc, paAlt, and paAr), and prokoniocortex (proA), as well as areas in the caudal and rostral parinsular location (reIt and paI). ${ }^{2}$ The anterior boundary of the auditory cortex was taken to be a vertical line drawn from the tip of the superior temporal sulcus to the lateral sulcus.

\section{Histology}

At the end of the experiment, each animal was euthanized with an overdose of sodium pentobarbital and then perfused transcardially with normal saline followed by $10 \%$ formalin. The head was then removed and placed in a $10 \%$ formalin solution. Seven days later, the head was mounted in a stereotaxic device, and vertical and horizontal pins were passed through the brain, the resulting tracks of which served as reference points for the cortical reconstruction. The brain was then removed, photographed, and placed in a $30 \%$ sucrose and $10 \%$ formalin solution and allowed to sink twice. It was then frozen and sectioned at $50 \mu \mathrm{m}$, and every tenth section was stained with cresyl violet.

${ }^{2}$ We have adopted the architectonic parcellations of Sanides (1972) and Pandya and Sanides (1973) merely for ease of exposition and as a guide to give the reader an appreciation of the location and extent of the lesions, and not to indicate that such areas, which are based on studies with rhesus monkeys, are necessarily present in the cebus monkey. Nevertheless, it is worthwhile to note that in terms of external morphology, the brains of the rhesus and cebus monkeys are remarkably similar. In addition, the placements of areas TA, TB, and TC in the cebus monkey (Bonin, 1938) are virtually identical to the placements of these areas in the rhesus monkey (Bonin and Bailey, 1947).

\section{RESULTS}

\section{Extent of lesions}

Cortical reconstructions and representative cross-sections for each monkey are shown in Figure 2. The lesions were largely as intended and generally included the rostral and caudal portions of the lower bank of the lateral sulcus, most of the ST cortex, sparing a small section in its mid-caudal extent, and about half of the upper bank of the superior temporal sulcus. The extent of the lesion corresponded closely with area TA of Bonin and Bailey (1947) and areas Ts1, Ts2, Ts3, and Tpt of Sanides (1972) and Pandya and Sanides (1973).

The area of cortex removed was measured from the flattened reconstructions, and the amount of damage sustained to the different components of the auditory cortex on the right and left sides is shown in Table 1 . The overall amount of auditory cortex removed was similar for all three animals (range, 49.8-54.3\%) as was the amount of cortex removed in the ST cortex (range, 82.3-90.1\%). For monkeys $\mathrm{B}$ and $\mathrm{T}$, the amount of cortex removed in the superior temporal sulcus $(44.2 \%$ and $39.3 \%)$ and the lateral sulcus $(30.7 \%$ and $27.8 \%)$ was similar. Relative to these two monkeys, monkey $\mathrm{M}$ had more cortex removed in the superior temporal sulcus $(58.3 \%)$ and less removed in the lateral sulcus $(14.7 \%)$. With the exception of monkey M, who sustained asymmetrical damage to the STS, there was very little difference between the right and left lesions.

\section{Visual DMS}

Neither unilateral nor bilateral ST cortex lesions had any effect on visual DMS performance. The performance of each monkey on the baseline $(0.5 \mathrm{sec})$ and retention $(0.5,4$, and $32 \mathrm{sec})$ DMS tasks preoperatively and after each operation is shown in Figure 3. Preoperatively, all three monkeys required only one session to attain criterion on the baseline DMS task. Postoperatively, the monkeys required between one and two sessions after the unilateral lesion, and only one session after the bilateral lesion, to achieve the criterion on the baseline DMS task.

The number of replications required to achieve stable retention DMS performance preoperatively and after each operation is also shown in Figure 3. Preoperatively, all three animals required only two replications to achieve stable retention gradients. Postoperatively, the monkeys required only one to two replications after the unilateral lesion and two to three replications after the bilateral lesion to achieve preoperative retention performance levels. ${ }^{3}$

\footnotetext{
${ }^{3}$ Monkey T, which required only two replications to achieve preoperative performance levels on the retention DMS task after the unilateral ST cortex lesion, was tested inadvertently for another two replications. Criterial performance was maintained on both of these replications.
} 


\section{B. "Flat map"}
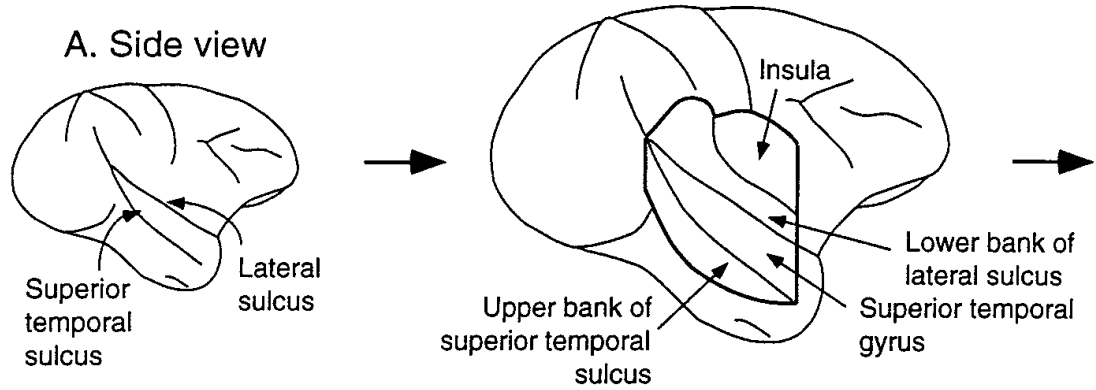

C. Intended lesion
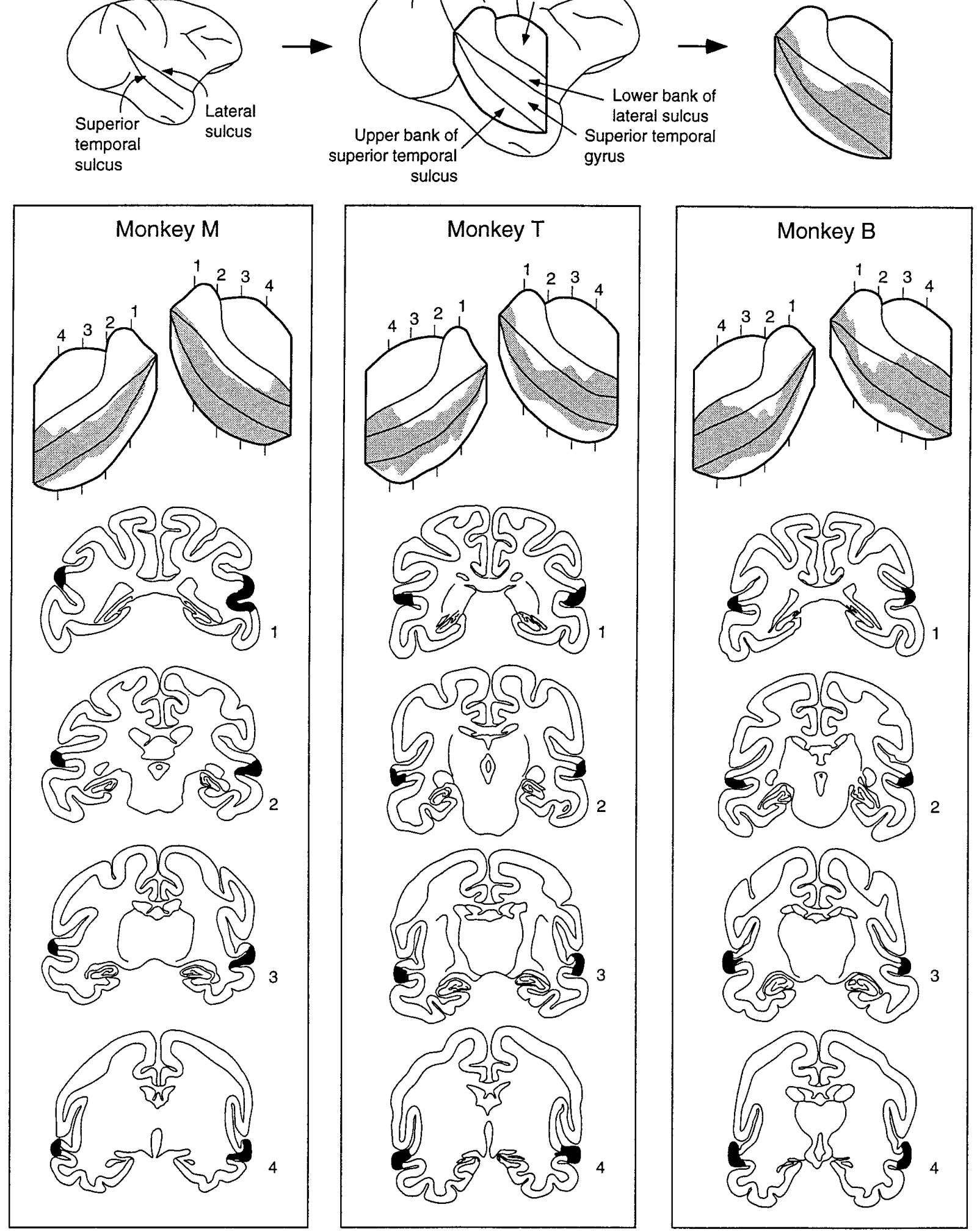

Figure 2. Flattened cortical reconstructions and representative cross-sections for monkeys M, T, and B. The shaded region represents the extent of the lesion. The top portion of the figure illustrates how the flattened reconstructions were generated. 


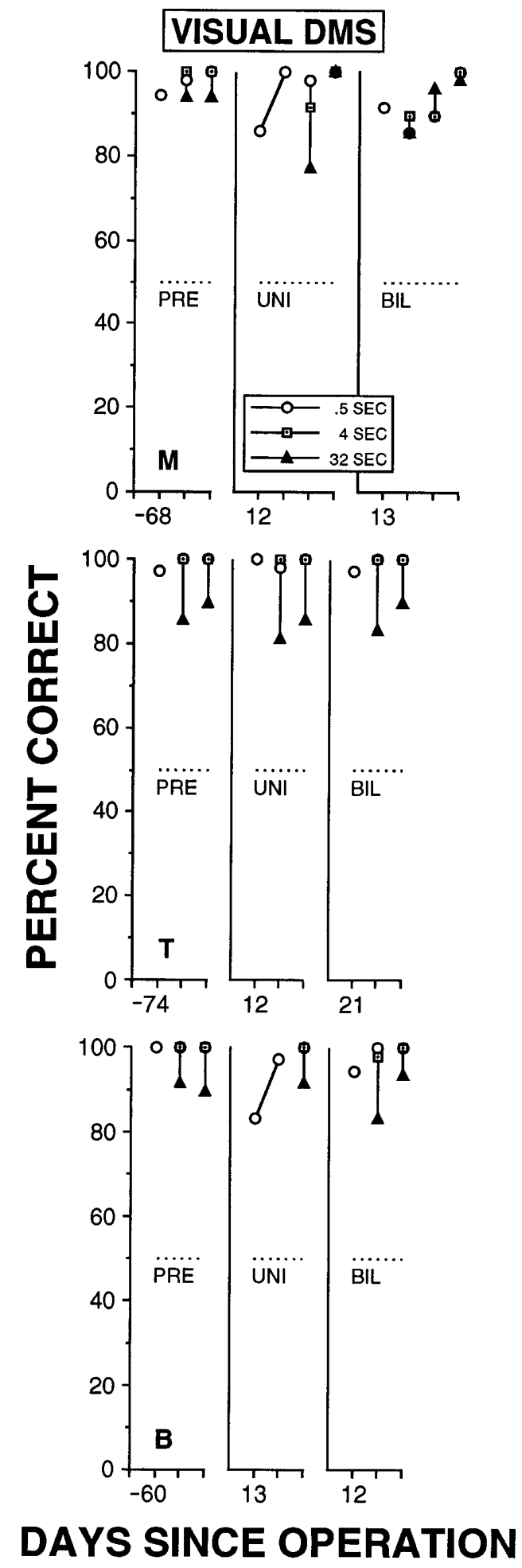

Figure 3. Performance on the visual DMS task preoperative (PRE) and after the first (UNI) and second (BIL) ST cortex lesions for monkeys M (top), T (middle), and B (bottom). Baseline DMS sessions, which used delays of $0.5 \mathrm{sec}$, are represented by a single circle. Retention DMS sessions, which used delays of $0.5,4$, and $32 \mathrm{sec}$, are represented by three symbols (circle, square, and triangle) connected by a vertical line. Each baseline DMS session is based on $1 \mathrm{~d}$ of testing, whereas each retention DMS session is based on the average performance over $4 \mathrm{~d}$ of testing. Shown at the bottom are the number of days after each operation when testing was initiated. The preoperative value, representing the number of days before the first operation when testing was initiated, is shown as a negative number. The dotted line represents chance levels of performance.

\section{Auditory DMS}

Unilateral lesions

The effects of ST cortex lesions on auditory DMS behavior is shown in Figure 4. Preoperatively, the monkeys required only one session to reach criterial levels on the baseline DMS task and two replications to generate stable preoperative retention gradients. After the unilateral ST cortex lesions, the monkeys required one to three sessions to satisfy the criterion on the baseline DMS task. Monkeys $\mathrm{M}$ and $\mathrm{T}$ then required five and six replications, respectively, to achieve preoperative retention DMS performance levels. Despite satisfying the criterion on the baseline DMS task, monkey B was unable to reach preoperative retention DMS levels after eight replications, and because there was little evidence of improvement, testing was terminated. A single-subject factorial ANOVA, with operation (2), delay (3), and days (4) as factors, applied to performance on the eighth replication revealed, as expected, a significant drop in performance compared with preoperative levels $\left[F_{(1,18)}=8.24, p<0.05\right]$. In addition to examining whether the operation affected the overall levels of DMS performance, we were also interested in evaluating whether the operation affected the rate of forgetting, i.e., whether there were any differences between the slopes of the preoperative and postoperative retention gradients. The operation $\times$ delay interaction, which compares the slopes of the preoperative and postoperative retention gradients, was not significant $(p=0.23)$, indicating that there were equal rates of forgetting before and after the operation.

The auditory DMS performance of monkey B was again evaluated $114 \mathrm{~d}$ after the first operation ( $48 \mathrm{~d}$ after the termination of the eighth replication). Despite requiring only one session to achieve criterial performance on the baseline DMS task, there was no evidence for improvement on the retention DMS task. In fact, performance on this retest replication was worse than performance on the first retention DMS replication after the operation. A single-subject factorial ANOVA applied to the retest replication data again revealed a significant drop in performance compared with preoperative levels $\left[F_{(1,18)}=27.51, p<0.01\right]$, and again no difference in the preoperative and postoperative rates of forgetting $(p=0.70)$.

\section{Bilateral lesions}

Ablation of the ST cortex in the remaining hemisphere resulted in severe auditory DMS impairments in all three monkeys. Although monkey $\mathrm{M}$ required only four sessions to relearn the baseline DMS task to criterion, she was unable to recover retention DMS performance to preoperative levels after six consecutive replications. A single-subject factorial ANOVA comparing the sixth replication and preoperative data indicated a significant overall impairment in auditory DMS performance $\left[F_{(1,18)}=45.88, p<\right.$ $0.01]$. The operation $\times$ delay interaction, however, was not significant $(p=0.59)$, indicating that the rate of forgetting after the second operation was similar to the preoperative rate of forgetting.

The fact that the short-delay performance averaged over the six consecutive replications was only $82 \%$ correct is particularly interesting given that monkey $\mathrm{M}$ averaged $91.7 \%$ correct on the baseline DMS criterial session immediately before DMS retention gradient testing. One possible explanation for the reason that short-delay performance suffered during retention DMS testing was that monkey M may have been operating in a "medium- and long-delay set" and that the rapid onset of the comparison stimulus on short-delay trials was unexpected, thus impairing perfor- 


\section{AUDITORY DMS}
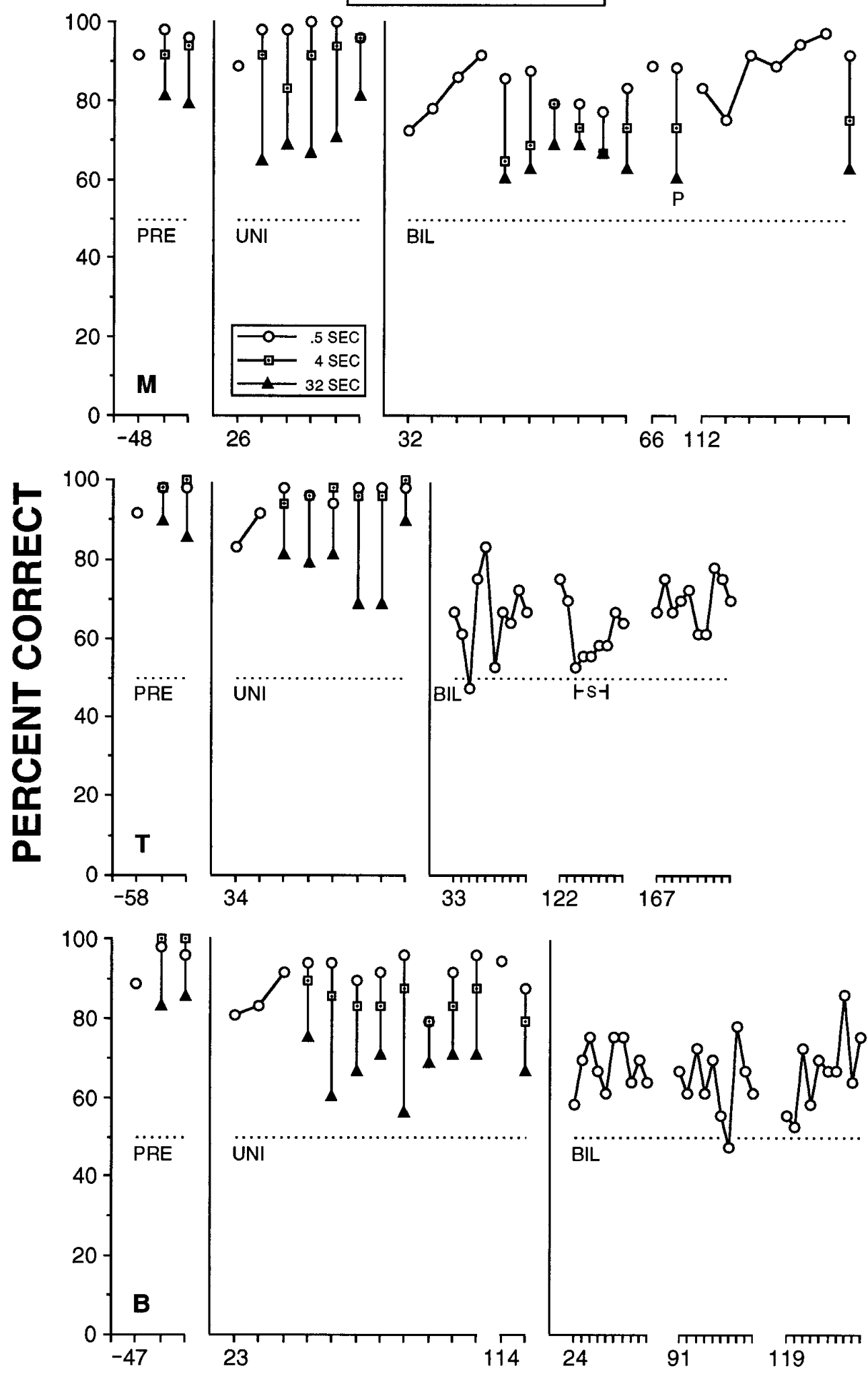

DAYS SINCE OPERATION
Figure 4. Performance on the auditory DMS task preoperatively (PRE) and after the first (UNI) and second (BIL) ST cortex lesions for monkeys $\mathrm{M}$ (top), $\mathrm{T}$ (middle), and B (bottom). Baseline DMS sessions, which used delays of $0.5 \mathrm{sec}$, are represented by a single circle. Retention DMS sessions, which used delays of 0.5 , 4 , and $32 \mathrm{sec}$, are represented by three symbols (circle, square, and triangle) connected by a vertical line. Each baseline DMS session is based on $1 \mathrm{~d}$ of testing, whereas each retention DMS session is based on the average performance over $4 \mathrm{~d}$ of testing. Shown at the bottom are the number of days after each operation when testing was initiated. The preoperative value, representing the number of days before the first operation when testing was initiated, is shown as a negative number. $P$ refers to proportional testing in which the proportion of short-delay trials was increased relative to mediumand long-delay trials; $S$ refers to testing with sine waves. The dotted line represents chance levels of performance. 
mance. To test this idea, after the completion of the sixth replication, monkey $\mathrm{M}$ was returned to the baseline DMS task and satisfied the baseline criterion in one session. Monkey $M$ was then tested on the retention DMS task. To encourage a "short-delay set," however, the constitution of a retention DMS session was changed from 12 trials at each delay to 24 short-, 6 medium-, and 6 long-delay trials. The results of changing the proportion of short, medium, and long delays are also presented in Figure 4 (indicated as $P$ ). To a certain degree, the manipulation had the predicted effect, with overall short-delay performance improving to a level higher than that reported in any of the previous six retention DMS replications [ 88.5 vs $82.0 \% ; t_{(5)}=3.93, p<0.05$ ]. Despite the elevation in performance at the short delay, however, there was no significant improvement in performance at the medium-delay interval ( 72.9 vs $70.9 \% ; p=0.38$ ), and performance at the long-delay period was significantly worse than in the six previous retention DMS replications [60.4 vs $65.0 \% ; t_{(5)}=$ $3.08, p<0.05]$. As a result of the improvement in performance at the short-delay interval, a single-subject factorial ANOVA revealed a significant operation $\times$ delay interaction $\left[F_{(2,18)}=3.95\right.$, $p<0.05$ ], suggesting a faster rate of forgetting postoperatively.

A final assessment of the retention DMS performance of monkey $\mathrm{M}$ was made $112 \mathrm{~d}$ after the second operation. In the hope of further elevating short-delay performance, monkey $M$ was first returned to the baseline auditory DMS task until she satisfied the criterion for four consecutive sessions. This difficult criterion was achieved in six sessions, with monkey $\mathrm{M}$ averaging 91.7, 88.9, 94.4, and $97.2 \%$ correct on the final four criterial sessions. After this training, monkey $\mathrm{M}$ was administered one final retention DMS replication and the results are again shown in Figure 4. Although short-delay performance rose to a level higher than that recorded for any other replication after the second operation $\left[91.7 \%, t_{(5)}=\right.$ $5.86, p<0.01$ ], there was no noticeable improvement in performance at the medium- $(p=0.11)$ or long-delay $(p=0.16)$ intervals. Despite the increase in performance at the short delay, a single-subject factorial ANOVA revealed that the operation $\times$ delay interaction was not significant $(p=0.27)$, again indicating similar rates of forgetting between the preoperative and this final retention DMS replication.

The auditory DMS deficits were even more pronounced for monkeys $\mathrm{B}$ and $\mathrm{T}$. The performances of each monkey on the baseline auditory DMS task during three different 10 -session periods after the second operation are shown in Figure 4. Despite various attempts at retraining the monkeys during a 4- to 5-month period, there was little indication that either animal would be able to relearn the baseline auditory DMS task. For example, the average performance of monkey T generated 167-176 d after the second operation showed very little change over performance generated 33-44 d postoperatively (69.4 vs $65.6 \%$, respectively, $p=0.32$ ). Likewise for monkey $\mathrm{B}$, a one-way ANOVA comparing baseline auditory DMS performance generated 24-33 d (67.8\%), 91-100 d (63.9\%), and 119-128 d (66.7\%) after the second operation indicated no significant change in level of performance $(p=0.57)$.

\section{Degree of impairments across delays}

To characterize further the auditory DMS impairments, we examined the effects that the lesions had on the short-, medium-, and long-delay periods by comparing the postoperative performance for each delay across all replications (omitting the criterial replication where applicable) to the average of the two corresponding preoperative delay values, using one-sample $t$ tests.
After the first operation, across the first four replications monkey M was impaired only at the long-delay interval $\left[t_{(3)}=9.30, p<\right.$ 0.01]. For monkey T, performance across the first five replications revealed impairments at both the medium- $\left[t_{(4)}=4.74, p<0.01\right]$ and long-delay $\left[t_{(4)}=4.00, p<0.05\right]$ intervals. Finally, across all nine replications, monkey $\mathrm{B}$ was impaired at the short- $\left[t_{(8)}=\right.$ $3.40, p<0.01]$, medium- $\left[t_{(8)}=13.03, p<0.01\right]$, and long-delay $\left[t_{(8)}=8.80, p<0.01\right]$ intervals. A similar examination made for monkey $\mathrm{M}$ after the second operation revealed that across all eight replications monkey $\mathrm{M}$ was impaired at the short- $\left[t_{(7)}=\right.$ 7.02, $p<0.01]$, medium- $\left[t_{(7)}=12.65, p<0.01\right]$, and long-delay $\left[t_{(7)}=13.13, p<0.01\right]$ periods.

Of the three animals after the first operation, monkey B was clearly the most impaired, followed by monkey $\mathrm{T}$, and then monkey M. Monkey B was not only impaired at the short-, medium-, and long-delay intervals, but within the time allotted for testing, monkey B was unable to achieve preoperative levels of performance at any of the three delay periods when tested on the retention DMS task. In this respect, the impairments of monkey B were quite similar to those of monkey $\mathrm{M}$ after the second operation, in that monkey $\mathrm{M}$ was also impaired at all three delay intervals, and within the time allotted for testing also seemed unable to achieve preoperative levels of performance at any of the three delay periods on the retention DMS task. (Note that both animals were able to attain preoperative levels on the baseline DMS task.) To examine whether there was any difference in the overall levels of impairment between monkeys B and M, we first computed the percentage drop in performance compared with preoperative levels for each delay for each postoperative replication. Planned comparisons based on a factorial ANOVA with subject (2: monkeys $\mathrm{B}$ and $\mathrm{T}$ ) and percentage drop at each delay (3: short, medium, and long) as factors revealed that monkey $\mathrm{M}$ was more impaired than monkey $\mathrm{B}$ at both the short-delay $\left[t_{(45)}=\right.$ $6.76, p<0.01]$ and medium-delay $\left[t_{(45)}=2.79, p<0.01\right]$ intervals, but not at the long-delay $(p=0.08)$ interval.

\section{Auditory DMS: other tests}

\section{Sine waves}

Because square waves are known to generate a large number of harmonics of the fundamental frequency, one possible explanation for the poor auditory DMS performance was that the lesions simply made the monkeys more sensitive to the interfering effects of the harmonics. To evaluate this possibility, monkey $\mathrm{T}$ was tested with the HT and LT stimuli delivered as sine waves rather than as square waves, and the results are shown in Figure 4 (marked as $S$ ). Testing with sine waves resulted in lower performance levels $(56.1 \%)$ than testing with square waves either immediately before $(72.2 \%)$ or after $(65.3 \%)$, a result that likely is attributable to a generalization decrement associated with the different quality of sound produced by the sine waves compared with square waves. Nevertheless, there was little indication, at least for monkey $\mathrm{T}$, that sensitivity to the harmonics generated by the square-wave sounds was responsible for the auditory DMS impairments.

\section{Transfer tests}

In the original auditory DMS study, D'Amato and Colombo (1985) reported that when monkeys B, M, and T learned to match-to-sample with a particular pair of auditory stimuli, they then transferred responding to novel pairs of auditory stimuli, indicating that they possessed a "matching concept" in the auditory modality. Given that monkey $\mathrm{M}$ was able to perform the 


\section{TRANSFER TEST}

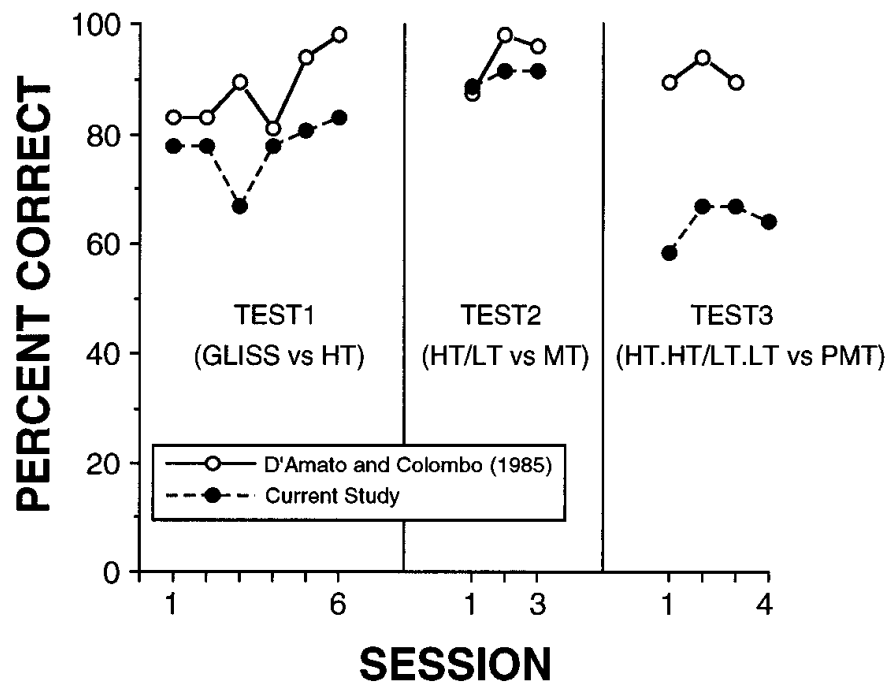

Figure 5. Transfer ability of monkey $\mathrm{M}$ to different stimuli after the second (bilateral) ST cortex lesion. Also shown are the transfer levels attained by monkey $\mathrm{M}$ in an earlier study. The acoustic stimuli of Test 1 were the HT $(3676 \mathrm{~Hz})$ and a Gliss tune ranging in frequency between 1437 and $8333 \mathrm{~Hz}$. In Test 2, the stimuli were an alternating $(0.7 \mathrm{~Hz}) \mathrm{HT}$ and LT $(243 \mathrm{~Hz})$ and a steady MT $(947 \mathrm{~Hz})$. The tempo difference between the stimuli used in Test 2 was eliminated in Test 3 by using alternating $(4 \mathrm{~Hz})$ doublets of the HT and LT and a pulsating $(4 \mathrm{~Hz})$ MT.

baseline auditory DMS task to criterial levels, we retested this animal on her ability to transfer responding to the same stimuli to which she showed high levels of transfer in the original study. The results of these transfer tests, along with her original transfer levels from the D'Amato and Colombo (1985) study, are shown in Figure 5.

For the first transfer test, the stimuli consisted of the standard HT stimulus and a "Gliss" tune composed of 11 monotonically ascending and 11 monotonically descending frequencies ranging between 1437 and $8333 \mathrm{~Hz}$. The duration of one complete playing of the Gliss tune was $1.5 \mathrm{sec}$. In the original study, monkey $\mathrm{M}$ averaged $88.2 \%$ correct over a six-session period, whereas in this current study she was able to average only $77.3 \%$ correct during a similar period $\left[t_{(10)}=3.04, p<0.05\right]$. The second transfer test used an alternating HT and LT $(0.7 \mathrm{~Hz})$ stimulus and a steady 947 $\mathrm{Hz}$ medium-frequency tone (MT) stimulus. In the original study, monkey $\mathrm{M}$ averaged $93.8 \%$ correct over a three-session period. In this current study, monkey $\mathrm{M}$ performed at comparable levels, averaging $90.7 \%$ correct over a similar period $(p=0.42)$. One explanation for this high level of transfer was that rather than relying on the frequency differences, monkey $\mathrm{M}$ was relying on the tempo differences between the alternating HT/LT and steady MT sounds. Thus, in the third transfer test, this tempo difference was eliminated by testing monkey $\mathrm{M}$ with a pulsed $(4 \mathrm{~Hz}) \mathrm{MT}$ and a pulsed $(4 \mathrm{~Hz}$ ) alternating series of HT and LT doublets (HT.HT/ LT.LT). With the tempo difference removed, over a three-session period monkey $\mathrm{M}$ performed much more poorly in this current study compared with the original transfer study (63.9 vs $91.0 \%$; $t_{(5)}$ $=10.41, p<0.01)$. In summary, the ability of monkey $\mathrm{M}$ to transfer responding to auditory-based features of stimuli to which she had shown high levels of transfer in a previous study was severely compromised after bilateral ST cortex lesions.

\section{Latency measures}

Although all of the animals were required to listen to the auditory sample stimulus for at least $2 \mathrm{sec}$, longer postoperative latencies might indicate, for example, that the ST cortex lesions affected the motivation levels of the monkeys. To evaluate this possibility, we computed the median latency to respond to the sample stimulus for each auditory baseline DMS session after the second operation, and we compared these values with the median latency of the two preoperative sessions using one-sample $t$ tests. There was no significant difference between the latency to respond to the auditory sample stimulus preoperatively or after the second ST cortex lesion for monkey M (2.30 vs $2.34 \mathrm{sec} ; p=0.10)$, monkey T (3.00 vs $3.06 \mathrm{sec} ; p=0.60)$, or monkey B (2.18 vs $2.37 \mathrm{sec} ; p=0.07)$. These results indicate that bilateral damage to the ST cortex did not alter the latency to respond to the sample stimulus on the baseline auditory DMS task.

\section{Auditory pattern discrimination}

The ability of all three monkeys to perform the auditory patterndiscrimination task, i.e., to discriminate between an ascending (2688-3012-3378-3788 Hz) and a descending (4808-4237-3788$3378 \mathrm{~Hz}$ ) series of tones, was also impaired by the bilateral ST cortex lesions. The number of sessions required to satisfy the criterion preoperatively and after the bilateral lesion are shown in Figure 6. Despite not having been exposed to the task for 196-206 $\mathrm{d}$, preoperatively all three monkeys relearned the pattern discrimination in two to three sessions. After bilateral ST cortex lesions, all three monkeys were impaired in their ability to perform the pattern-discrimination task. Monkey $M$ required 13 sessions to relearn the task and showed a steady improvement in performance over the course of that period. In contrast, monkeys B and $\mathrm{T}$ showed very little evidence of improvement, averaging $54.2 \%$ correct and 54\% correct over the course of 8 and 15 sessions of testing, respectively.

\section{Frequency discrimination}

A critical question is whether the monkeys, in particular monkeys B and T, could discriminate the HT $(3676 \mathrm{~Hz})$ and LT $(243 \mathrm{~Hz})$ sounds used in the auditory DMS task. To examine this issue, all three monkeys were first tested on their ability to discriminate between two sounds in the HT frequency range: $4808 \mathrm{~Hz}(\mathrm{~S}+)$ and $2688 \mathrm{~Hz}(\mathrm{~S}-)$. The results are shown in Figure 7, top. Monkeys $\mathrm{M}$ and $\mathrm{T}$ required one and seven sessions, respectively, to satisfy the criterion of one session with at least 43 out of 48 $(89.6 \%)$ correct responses. Monkey B, on the other hand, showed very little evidence of improvement over the course of the first 12 sessions, averaging $57.8 \%$ correct. When tested again $55 \mathrm{~d}$ later, monkey B was still unable to satisfy the criterion despite 15 sessions of testing, although overall performance did improve to $72.9 \%$ correct.

The monkeys were also trained to discriminate between two sounds in the LT frequency range, $613 \mathrm{~Hz}(\mathrm{~S}+)$ and $306 \mathrm{~Hz}(\mathrm{~S}-)$, and the results are shown in Figure 7, bottom. Monkeys $\mathrm{M}$ and $\mathrm{T}$ required 5 and 15 sessions, respectively, to learn the task to criterion. Paralleling the deficits experienced with the highfrequency sounds, monkey B had difficulty discriminating between the two low-frequency sounds, but was able to average $74.2 \%$ correct over the last 10 of 20 sessions when testing was terminated because of time constraints.

\section{Serial-order task}

Neither unilateral nor bilateral ST cortex lesions had any effect on performance of the visual serial-order task. Preoperatively the 


\section{PATTERN DISCRIMINATION}
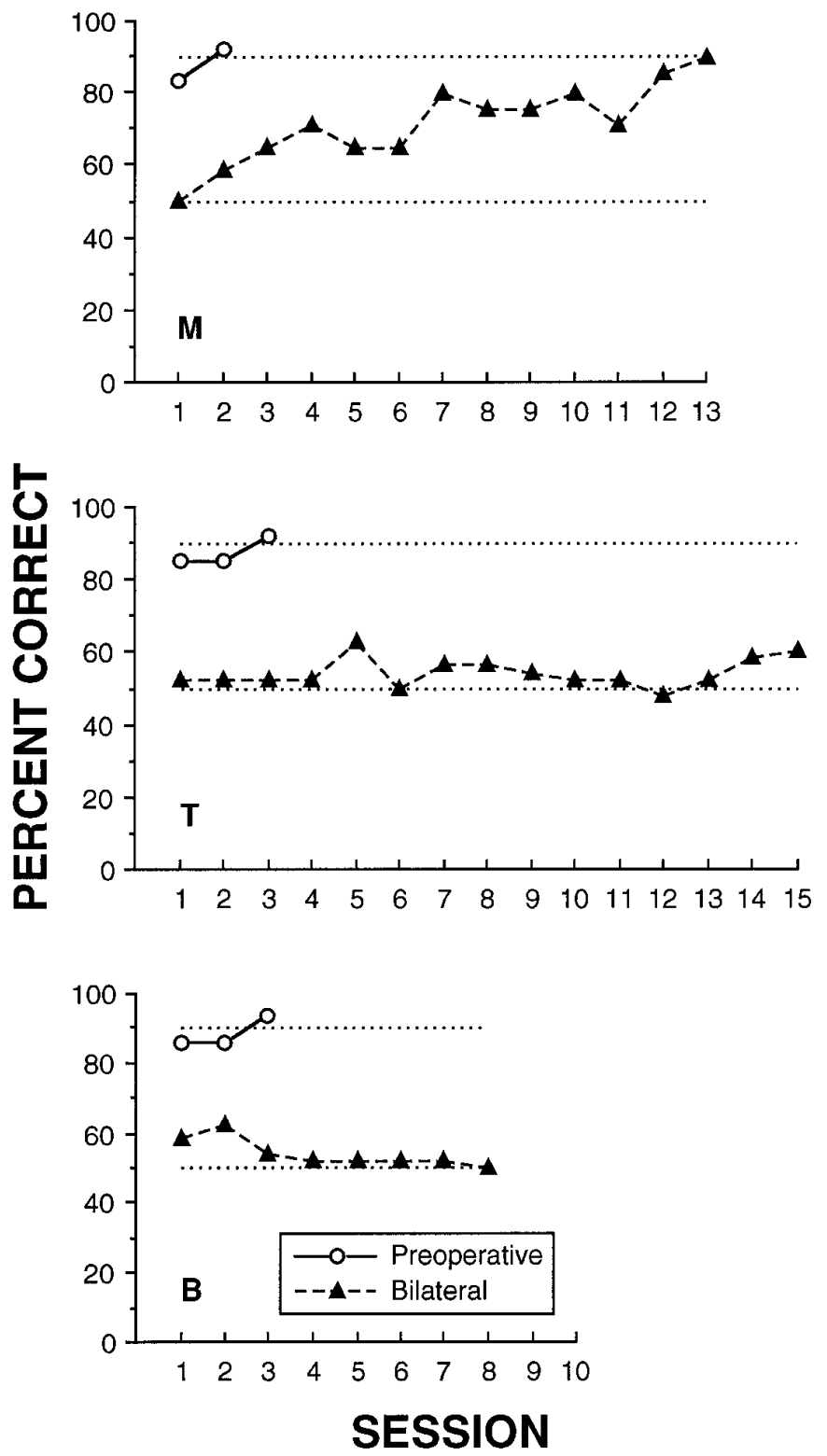

Figure 6. Performance on the pattern-discrimination task preoperatively and after the second (bilateral) ST cortex lesion for monkeys M, T, and B. The top and bottom dotted lines represent criterial and chance levels of performance, respectively.

monkeys required between two and three sessions to achieve criterion on the baseline ABCD serial-order task. Postoperatively, the monkeys required between three and seven sessions after the unilateral lesions and between two and three sessions after the bilateral lesions to achieve criterial levels on the ABCD serialorder task.

In a previous study, D'Amato and Colombo (1988b) reported that latency to respond to the first item of a test pair increased as a function of the position of the item in the series (first-item latency effect) and that the latency to respond to the second item of a pair increased as a function of the number of missing items separating the first and second items (second-item latency effect). The first-item and missing-item latencies generated preopera-
FREQUENCY DISCRIMINATION

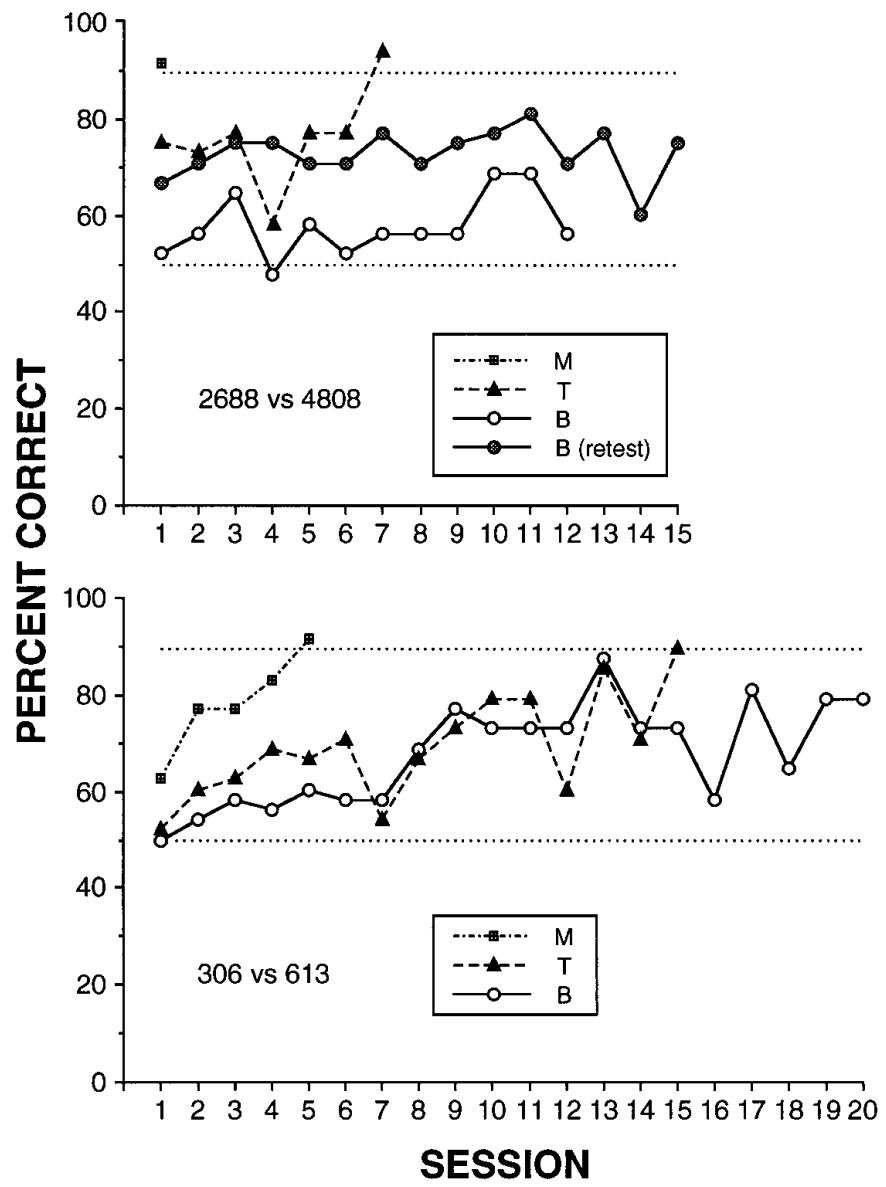

Figure 7. Performance on the high-range $(2688$ vs $4808 \mathrm{~Hz})$ and lowrange (306 vs $613 \mathrm{~Hz}$ ) frequency-discrimination tasks preoperatively and after the second (bilateral) ST cortex lesion for monkeys M, T, and B. The top and bottom dotted lines represent criterial and chance levels of performance, respectively.

tively and after each operation are shown in Figure 8. A withinsubjects ANOVA was applied to the first-item and missing-item latency data. Planned polynomial contrasts revealed a significant linear trend for the first-item latencies $\left[F_{(1,4)}=9.82, p<0.05\right]$ and missing-item latencies $\left[F_{(1,4)}=12.07, p<0.05\right]$, indicating that the first-item and missing-item latency effects noted in the original D'Amato and Colombo (1988b) study were apparent in the current study. Although there were changes in the speed with which the task was solved, which is common with repeated exposure to the pairwise test (D'Amato and Colombo, 1988b; Colombo et al., 1993), there was no indication that either unilateral or bilateral ST cortex lesions affected the overall pattern of the first-item ( $p=$ 0.77 ) or missing-item ( $p=0.07)$ latency effects.

\section{DISCUSSION}

\section{Summary of findings}

Neither unilateral nor bilateral ST cortex lesions had any effect on visual DMS or visual serial-order behavior. In contrast, the unilateral and bilateral ST cortex lesions resulted in moderate and severe impairments, respectively, on the auditory DMS task. Preoperatively the three monkeys required only one session to attain criterial levels on the baseline DMS task and only two replications to generate stable retention gradients. After the unilateral ST 

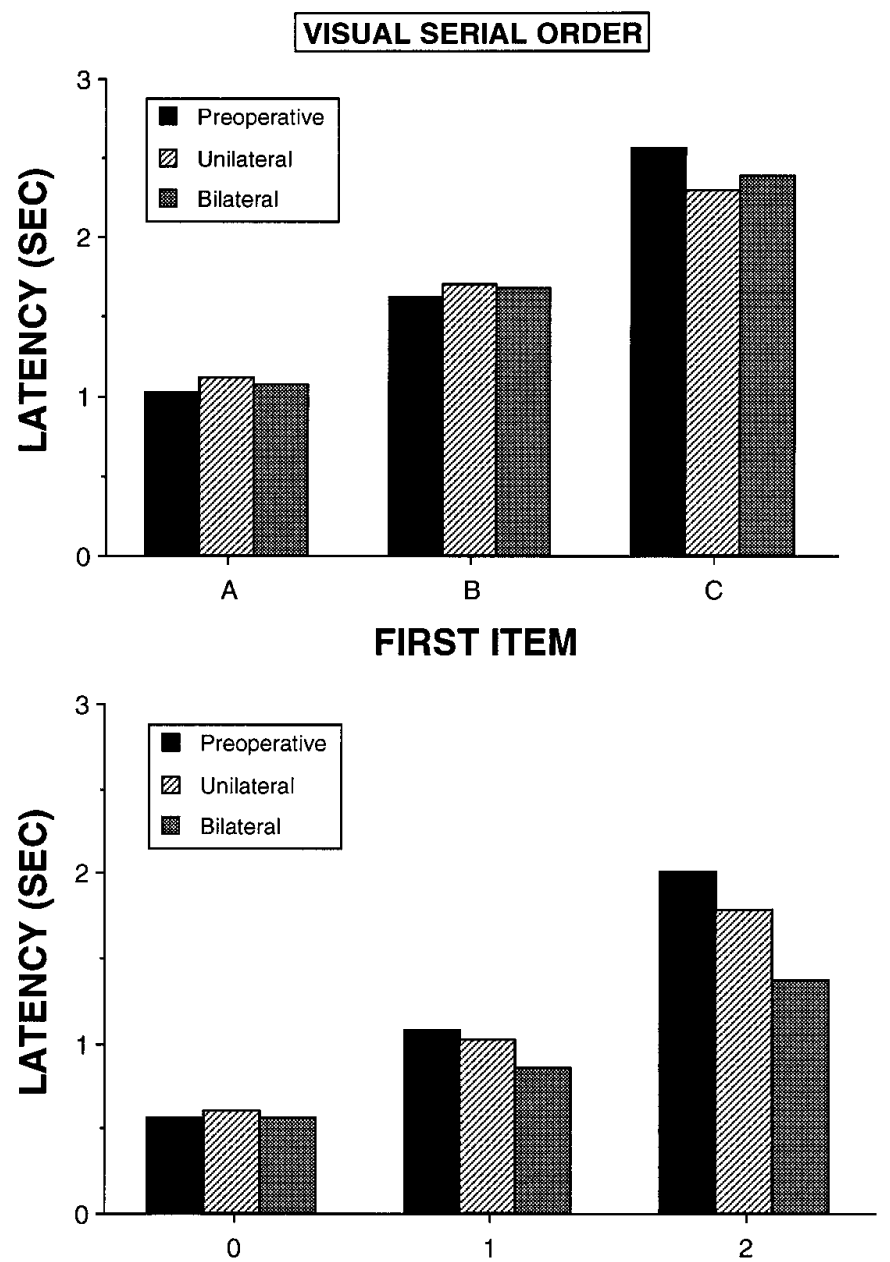

NUMBER OF MISSING ITEMS

Figure 8. The first-item latency effect (top) and missing-items latency effect (bottom) generated preoperatively and after the first (unilateral) and second (bilateral) ST cortex lesions. In the top, the response latency is to the first item of a test pair as a function of whether the first item is stimulus $\mathrm{A}$ (pairs $\mathrm{AB}, \mathrm{AC}$, and $\mathrm{AD}$ ), B (pairs $\mathrm{BC}$ and $\mathrm{BD}$ ), or $\mathrm{C}$ (pair $\mathrm{CD}$ ). In the bottom, the response latency is to the second item of a test pair as a function of whether the number of missing items is 0 (pairs $\mathrm{AB}, \mathrm{BC}$, and $\mathrm{CD}$ ), 1 (pairs $\mathrm{AC}$ and $\mathrm{BD}$ ), or 2 (pair $\mathrm{AD}$ ). The figures are collapsed across all three monkeys and across the two preoperative, two unilateral, and two bilateral test sessions.

cortex lesion, monkeys $\mathrm{M}$ and $\mathrm{T}$ required between one and two sessions, respectively, to attain criterion on the baseline DMS task, and five and six replications, respectively, to attain preoperative levels on the retention DMS task. Although monkey B was able to attain criterion on the baseline DMS task in three sessions, he was unable to attain preoperative levels on the retention DMS task despite testing over a period of $\sim 3$ months. Paralleling the deficits in relearning the retention DMS task, relative to preoperative performance levels monkey $\mathrm{M}$ was impaired at only the short-delay interval, monkey $\mathrm{T}$ was impaired at both the shortand medium-delay intervals, and monkey B was impaired at all three delay intervals.

After the bilateral lesions, only monkey $\mathrm{M}$ was able to attain criterial levels on the baseline DMS task. Despite a number of attempts over a period of $\sim 4$ months, however, there was no indication that monkey $\mathrm{M}$ was able to attain preoperative levels on the retention DMS task at any of the three delay intervals. The situation for monkeys B and $\mathrm{T}$ was even more serious. Despite testing over a period of $\sim 4-5$ months, neither animal was able to attain criterial levels on the baseline DMS task. Although all three monkeys were impaired in performing the auditory DMS task with two stimuli separated by almost four octaves, monkey $\mathrm{M}$ was able to discriminate with little difficulty between two highfrequency sounds separated by only one octave and between two low-frequency sounds also separated by only one octave; monkey $\mathrm{T}$ was able to attain criterial levels on both frequency discriminations but required substantially more sessions to do so than monkey M; and monkey B was unable to discriminate between the high- and low-frequency stimuli to criterial levels but nevertheless was significantly above chance levels.

In addition to impairments on the auditory DMS task, after the bilateral ST cortex lesions all three monkeys were also impaired in their ability to relearn the auditory pattern-discrimination task. Despite not having been exposed to the auditory patterndiscrimination task for $>6$ months, preoperatively the monkey required only two to three sessions to relearn the task to criterial levels. After the bilateral lesions, monkey $\mathrm{M}$ was able to relearn the task to criterial levels, showing a steady improvement over the 13 sessions required to do so. Neither monkey B nor monkey $\mathrm{T}$ showed any improvement in performing the auditory patterndiscrimination task over 8 and 15 sessions, respectively, and their performance during these periods was virtually at chance levels.

The auditory DMS and pattern-discrimination deficits were apparent despite the fact that preoperatively all three monkeys were extensively overtrained on both these tasks. This was particularly so for the auditory DMS task, where all three animals were trained for a period of 3-4 years before the start of the current experiment. During that time, they accumulated $\sim 11,000-19,000$ auditory DMS trials, with delays ranging from 0 to $32 \mathrm{sec}$. Yet despite this extensive overtraining, and despite the fact that overtraining has often been shown to reduce the effects of lesions to IT cortex (Chow and Survis, 1958; Orbach and Fantz, 1958), the ST cortex lesions resulted in severe impairments in auditory DMS behavior. In contrast, the monkeys received much less training on the visual DMS task, with delays ranging from 0 to $32 \mathrm{sec}(\sim 3000-$ 8000 trials), yet visual DMS behavior was not affected in the least by the ST cortex lesions.

\section{Relation between lesion site and behavioral deficits}

It was the intention in this study to lesion auditory association cortex (area TA), sparing both primary auditory koniocortex (area TC) and secondary auditory parakoniocortex (area TB). In terms of the architectonic parcellation scheme of Sanides (1972) and Pandya and Sanides (1973), the intention was to lesion areas Ts1, Ts2, Ts3, and Tpt on the ST cortex, as well as the extensions of these regions into the lateral sulcus and superior temporal sulcus. For the most part, the actual lesions were very close to the intended lesion. Thus for all three animals, the lesions included the rostral and caudal ends of the lower bank of the lateral sulcus, most of the ST cortex sparing a portion in its mid-caudal extent, and approximately half of the upper bank of the superior temporal sulcus.

Despite general similarities in the pattern of the lesions, it was clear after the second operation that the three monkeys were impaired to differing degrees on the baseline auditory DMS, pattern-discrimination, and frequency-discrimination tasks. Monkey $\mathrm{M}$, for example, was the least impaired of the three subjects, being able to relearn the baseline auditory DMS and patterndiscrimination tasks as well as rapidly acquiring the frequencydiscrimination task. In contrast, monkeys $\mathrm{B}$ and $\mathrm{T}$ both showed 
little indication of being able to relearn the baseline auditory DMS or pattern-discrimination tasks, and relative to monkey M, both were certainly more impaired on the frequencydiscrimination task.

The differing degree of behavioral impairments noted in the three monkeys does not seem to be related to the amount of damage sustained by either the ST cortex or the superior temporal sulcus. Indeed, if anything, monkey $M$ sustained more damage to these two regions than did monkeys B or T, yet was least impaired on the auditory tasks. Rather, the degree of behavioral impairments seems to correlate with the amount of damage sustained to the lower bank of the lateral sulcus, with monkeys $\mathrm{B}$ and $\mathrm{T}$ receiving about twice as much damage to this region as monkey M. In addition, the critical area within the lateral sulcus seems to be the anterior rather than the posterior region. The reason is that monkeys $\mathrm{B}$ and $\mathrm{T}$, who were most impaired on the auditory tasks, both received more than twice as much damage to the anterior lateral sulcus ( 24.5 and $24.9 \%$, respectively) compared with monkey $\mathrm{M}(11.4 \%)$. In contrast, although monkey $\mathrm{B}$ received the largest amount of damage to the posterior lateral sulcus $(6.2 \%)$, there was little difference between the amount of damage sustained to this region for monkeys $\mathrm{M}$ and $\mathrm{T}$ (3.3 and 2.9\%, respectively).

\section{Hemispheric asymmetries}

Both behavioral (Petersen et al., 1978; Hauser and Andersson, 1994) and neural (Dewson, 1977; Heffner and Heffner, 1984, 1986a; Gaffan and Harrison, 1991) studies have indicated that the left ST cortex in the monkey brain is specialized for processing auditory information. It is interesting to note, therefore, that after the first operation, monkey $\mathrm{B}$ and to a lesser extent monkey $\mathrm{T}$, both of whom sustained left ST cortex lesions, were more impaired on the auditory DMS task than monkey M, who sustained a right ST cortex lesion. Although this might be viewed as support for the notion of hemispheric asymmetry of auditory function, perhaps a more parsimonious interpretation in the current situation is that the degree of impairment after the unilateral lesion was related to the amount of damage sustained in the lower bank of the lateral sulcus $(24.7,22.6$, and $14.9 \%$, for monkeys B, T, and $\mathrm{M}$, respectively). This is not to say that the auditory cortex is not lateralized along the dimensions specified by others, but merely that in this current situation asymmetry of function is not the only or most parsimonious interpretation of the data.

Issues of asymmetry aside, that monkey B was impaired on the auditory DMS task after the unilateral lesion merits further consideration. Of particular interest is the fact that the auditory DMS impairments sustained by monkey B after the unilateral lesions were similar to the auditory DMS impairments sustained by monkey $\mathrm{M}$ after the bilateral lesions. For example, although both subjects were able to achieve criterial levels of performance on the baseline $(0.5 \mathrm{sec})$ DMS task, both experienced difficulty at all three delay intervals on the retention DMS task, and indeed within the time allotted for testing, both were unable to recover retention DMS performance to preoperative levels. Despite these similarities, it was clear that after the second operation monkey $\mathrm{M}$ was quantitatively more impaired at the short- and medium-delay intervals than monkey B after the first operation. Although scant evidence exists, similar suggestions from the IT cortex lesion literature indicate that unilateral disruption of IT cortex does impair visual DMS performance (Kovner and Stamm, 1972), and these impairments are generally less severe than those observed after bilateral disruption to IT cortex (Fuster et al., 1981).

\section{Comparison with previous auditory memory studies}

In contrast to previous studies that have failed to find any effects of ST cortex lesions on auditory memory (Dewson et al., 1970; Cowey and Dewson, 1972; Cowey and Weiskrantz, 1976; Dewson, 1977, 1978, 1979; Pratt and Iversen, 1978; Costalupes, 1984), the results of this current study indicate that when monkeys are trained on a DMS task using auditory sample, and auditory comparison stimuli in particular, then bilateral ST cortex lesions result in severe impairments in auditory memory. It seems likely, therefore, that the failure of previous attempts to obtain auditory memory deficits after ST lesions might indeed have been because the monkeys were engaging in prospective processing, i.e., remembering visual and spatial rather than auditory information during the delay period of the auditory-visual and auditoryspatial DMS tasks, respectively.

That monkeys would recode auditory information into a visual code on an auditory-visual DMS task is consistent with a number of studies showing that animals tend to display superior retention when information is processed through their dominant sensory modality (Herman, 1980; Wallace et al., 1980; Herman and Forestell, 1985; Colombo and D'Amato, 1986). In view of this modality bias, it seems likely that when exposed to an auditory-visual DMS task, the visually dominant monkey would recode the auditory sample information into the correct visual comparison stimulus and remember visual information throughout the delay period. In support of this, we have shown that auditory-visual DMS performance in monkeys is much more sensitive to delay-interval visual interference than to delay-interval auditory interference, an outcome that can be explained only if the monkeys were converting the auditory sample information into a visual code and remembering visual information throughout the delay period (Colombo and Graziano, 1994).

Although it is unclear from the perspective of a modality bias the weight that a monkey would assign to auditory and spatial information, the fact that monkeys learn spatial tasks with apparently greater ease than they learn auditory tasks also would seem to indicate a preference for processing spatial over auditory information. Regardless of whether monkeys would prefer to recode auditory information into a spatial code, the auditory-spatial DMS task presents a second interpretational problem beyond that of processing strategy. That is, not only is it possible for monkeys to bypass auditory memory by prospectively processing spatial information on an auditory-spatial DMS task, but the nature of a spatial response is such that an animal could bypass memory altogether by adopting a correct body orientation during the delay period (Hunter, 1913; Kojima, 1980). For example, in the Pratt and Iversen study (1978), the required response was to press either a right or a left lever after a delay period. To solve such a task without using memory, the subjects need only to have heard the auditory stimulus and then immediately situate themselves in front of the proper response key during the delay period. In summary, the adoption of either a prospective strategy or a body orientation strategy would make it such that ST cortex lesions would have little or no effect on auditory-spatial DMS performance.

\section{Comparison with previous auditory pattern-discrimination studies}

In contrast to previous pattern-discrimination/lesion studies, which typically included damage to primary auditory koniocortex, the results from this current study indicate that in addition to causing impairments in auditory memory, ST cortex lesions that 
seemed to spare primary auditory koniocortex also caused impairments in the ability to discriminate between an ascending and a descending pattern of tones. These deficits were apparent despite the fact that the monkeys received considerable preoperative experience discriminating between the two auditory patterns, and despite the fact that preoperatively they were able to relearn the task within two to three sessions even though they had not been exposed to the task for $>6$ months.

Although the results of this current study suggest that damage sparing auditory koniocortex can result in auditory patterndiscrimination impairments, the extent to which the patterndiscrimination deficits arise from damage restricted to ST cortex alone as opposed to encroachment onto parakoniocortical regions in the anterior lower bank of the lateral sulcus remains unclear. Wegener (1976), for example, has shown that lesions of the anterior lower bank of the lateral sulcus (area Ts3 and parakoniocortical areas paAr and $\mathrm{PaI}$ ) also produce auditory patterndiscrimination impairments in monkeys. Although it is difficult to determine with reference to the architectonic parcellation scheme of Sanides (1972) and Pandya and Sanides (1973) the extent to which the current lesions encroached onto parakoniocortical areas, it is clear that monkeys $\mathrm{B}$ and $\mathrm{T}$, who were most impaired on the auditory pattern-discrimination task, also received greater amounts of damage to the anterior portion of the lower bank of the lateral sulcus than monkey M, who was least impaired on the auditory pattern-discrimination task.

It is worth noting that although the auditory patterndiscrimination task could have been solved on the basis of the ascending (2688-3012-3378-3788 Hz) and descending (4808$4237-3788-3378 \mathrm{~Hz}$ ) overall pattern of the sounds, it could also have been solved on the basis of the fact that the first two notes of the ascending series were different from and did not overlap with the first two notes of the descending series, thus reducing the task to a frequency discrimination. Likewise, although there was complete overlap of the notes used in the pattern-discrimination studies of Neff and colleagues (Jerison and Neff, 1953; Neff, 1961a, 1961b) $(800-1000-800 \mathrm{~Hz}$ vs $1000-800-1000 \mathrm{~Hz})$ and the Pratt and Iversen (1978) study (2400-300-2400 Hz vs 300-2400$300 \mathrm{~Hz}$ ), the pattern discriminations could have been based on the first note of the two sounds, which were always different, again reducing the task to a frequency discrimination. In fact, there is little evidence that monkeys are able to extract pattern from nonspecies-specific sounds and often rely on local frequency differences in the patterns to solve "pattern"-discrimination tasks (D'Amato and Salmon, 1982, 1984; D'Amato and Colombo, 1988a). If this is true, then the results of this current study and those of earlier pattern-discrimination/lesion studies may indicate that ST cortex is not so much critical for pattern discriminations as for any complex frequency discrimination.

\section{The mechanisms disrupted by ST cortex lesions}

A number of factors can be ruled out as the basis for the auditory DMS impairments observed in the present study. For example, two variables that are known to affect the behavior of animals are their motivation (Hull, 1943) and attention levels (Sutherland and Mackintosh, 1971; Wagner, 1981). The modality-specific nature of the impairments, however, rules out the possibility that general motivational factors accounted for the auditory DMS deficits. Furthermore, because the response latencies to initiate a trial did not change from preoperative levels, it is difficult to argue that the ST cortex lesions impaired the ability of the monkeys to attend to the auditory stimuli.
Another factor that could account for the auditory DMS impairments is a potential loss of hearing caused by the ST cortex lesions. In a series of elegant experiments, Heffner and Heffner (1986b, 1990) showed that damage to the auditory cortex in monkeys can result in a permanent hearing loss $(\sim 40 \mathrm{~dB})$ in the $250 \mathrm{~Hz}$ to $16 \mathrm{kHz}$ range, a frequency range that encompasses the HT and LT stimuli used in the auditory DMS task. It seems unlikely, however, that the ST cortex lesions could have caused a hearing loss sufficient to account for the auditory memory impairments in the present study. First, the hearing losses characterized by Heffner and Heffner (1986b, 1990) seem to occur only after extensive removal of primary auditory cortex (Heffner and Heffner, 1989). In addition, the results from a number of other studies also indicate that auditory thresholds in the 300-900 Hz (Iversen and Mishkin, 1973), 500-1000 Hz (Massopust et al., 1967), and $4000-5000 \mathrm{~Hz}$ (Massopust et al., 1970) frequency ranges are not impaired by damage restricted to the ST cortex. It seems likely, therefore, that ST cortex lesions produce little, if any, hearing loss.

\section{Auditory memory failure?}

One possibility is that the ST cortex lesions have impaired auditory memory. Such a statement, however, carries with it the implication that the deficit is in simply remembering auditory information, and that other aspects of auditory processing are intact. The results of this study suggest that more than just the ability to remember auditory information was affected by the ST cortex lesions. For example, note some of the auditory DMS findings shown with monkey $\mathrm{M}$ after the second operation. A pure memory disorder is difficult to reconcile with the fact that (1) in most cases there was little difference between the preoperative and postoperative rates of forgetting, indicating that the lesions impaired performance equally across all three delay periods; (2) performance at the $0.5 \mathrm{sec}$ delay period during retention DMS testing with delays of $0.5,4$, and $32 \mathrm{sec}$ was significantly lower than performance at the $0.5 \mathrm{sec}$ delay period during baseline DMS testing with delays of only $0.5 \mathrm{sec}$; and (3) there was little evidence of transfer to stimuli to which there were high levels of transfer in a previous study.

There are other lines of evidence that also argue against the suggestion that the ST cortex lesions affected just auditory memory. For example, all three monkeys were impaired to some degree on the auditory pattern-discrimination task. Although one might argue that the ability to distinguish between an ascending and a descending series of tones requires retention of each element in the series and hence the use of auditory memory, monkeys $\mathrm{B}$ and $\mathrm{T}$ were impaired on the high- and low-range frequency discriminations, which certainly rely less on the type of memory that might be required to solve the pattern-discrimination task. In sum, there are a number of lines of evidence, which suggests that the ST cortex lesions caused more than simply an inability to remember auditory information.

A similar argument that more than just memory is affected can also be made with respect to the effects of IT cortex lesions on visual DMS behavior in monkeys. Indeed, in many respects the effects of ST and IT cortex lesions on auditory DMS and visual DMS behavior, respectively, are quite similar. For example, the performance drop at all delay periods noted in this present study, particularly the shortest delay, is exactly the same result that appears in IT cortex lesion studies, and is apparent regardless of whether the visual DMS task is trial-repetitive or trial-unique, or matching or nonmatching, and is independent of whether the stimuli are colors, two-dimensional forms or three-dimensional 
objects (Dean, 1974, 1976, 1982; Sahgal and Iversen, 1978a,b; Fuster et al., 1981; Horel and Pytko, 1982; Mishkin, 1982; Horel et al., 1984; but see Eacott et al., 1994, for evidence that rhinal cortex lesions, i.e., lesions to anterior and ventral IT cortex, differentially affect trial-unique and trial-repetitive visual DMS tasks and may not cause impairments at the shortest delay period). In fact, IT cortex lesions even impair performance under conditions of simultaneous matching in which the sample and comparison stimuli are present at the same time on the displays (Ibuka et al., 1974; Sahgal and Iversen, 1978a,b; Fuster et al., 1981).

\section{Levels of processing failure?}

A second possible explanation of the auditory DMS deficits noted in the present study is that the ST cortex lesions caused impairments in the ability to process auditory information and that the auditory memory impairments are a product of this processing failure. Such a mechanism would be consistent with what has been referred to in the human literature as a "levels of processing" effect. This view, originally espoused by Craik and Lockhart (1972), proposes that memory is an emergent quality of information processing and that the level at which information is processed will determine how long information is remembered. Given that the degree of perceptual impairment would constrain the level at which information can be processed, the degree of perceptual impairment therefore would determine the degree of memory impairment. It is clear that in the current study the ST cortex lesions caused some degree of auditory perceptual impairments. More to the point, the degree of memory loss across the three animals was clearly related to their degree of perceptual loss. For example, monkey $\mathrm{M}$ exhibited the least impairment on the frequency and pattern-discrimination tasks and also the least impairment on the auditory DMS task, whereas monkeys B and T exhibited much greater frequency and pattern-discrimination impairments and also much greater auditory DMS impairments.

A similar "levels of processing" argument can account for the effects of IT cortex lesions on visual memory in monkeys. For example, it is well established that IT cortex lesions do not produce visual field defects (Cowey and Weiskrantz, 1963, 1967) or impair basic visual-sensory capacities (Weiskrantz and Cowey, 1963; Symmes, 1965; Bender, 1973; Bender and Gross, 1981). Does this mean that IT animals with visual memory impairments are normal in all other aspects of visual perception and that they experience the visual world in the same manner as unoperated animals? Not quite. In addition to impairments in color vision (Heywood et al., 1995), studies of equivalence behavior in which monkeys are trained with one class of stimuli and then tested with slightly altered versions of that same class have shown that monkeys with IT cortex lesions do not rely on the same cues for discriminating stimuli as normal animals do (Butter et al., 1965; Butter and Gekoski, 1966; Butter, 1968; Butter and Doehrman, 1968; Butter and Hirtzel, 1970; Iwai, 1985). It is possible, therefore, that the visual memory impairments that accompany IT cortex lesions might also be a product of disruptions in the processing of visual information. ${ }^{4}$

\footnotetext{
${ }^{4}$ Inferior temporal cortex is usually divided into two major subdivisions, areas TEO (more posterior) and TE (more anterior), which differ in their architecture (Bonin and Bailey, 1947; Boussaoud et al., 1991), anatomical connections (Webster et al., 1991; Gross et al., 1993; Webster et al., 1994, 1995), visuotopic organization (Gross et al., 1969; Desimone and Gross, 1979; Boussaoud et al., 1991; Gross et al., 1993), single neuron properties (Iwai, 1985; Tanaka et al., 1991; Gross et al., 1993), and development (Webster et al., 1991; Gross and Rodman, 1992; Rodman, 1994). These regions also differ in terms of the behavioral effects after lesions, with damage to area
}

The idea that perceptual problems might underlie the memory impairments that follow IT cortex lesions has been expressed by a number of investigators. For example, Dean $(1976,1982)$ (see also Wilson, 1968) has suggested that IT lesions in monkeys result in impairments in the ability to categorize visual information effectively such that the resulting categories are either fewer or of lesser precision. Although the mechanisms by which perceptual impairments may result in memory impairments are far from clear, the available evidence from IT cortex studies and this study, as well as emerging evidence from auditory studies in humans (Zatorre, 1985; Samson and Zatorre, 1992; Peretz et al., 1994; Zatorre et al., 1994), indicates that it may be difficult to obtain memory deficits in the absence of perceptual deficits from lesions of higher-order visual and auditory cortical areas.

\section{Summary and conclusions}

Anatomically, the visual and auditory systems are similar in terms of both their internal and external projection patterns. For example, both systems have quite similar outward sequentially organized projections that originate and terminate in specific cortical layers (Pandya and Yeterian, 1985). In addition, both system have similar extrinsic projection patterns to frontal, limbic, and parietal cortex (Pandya and Kuypers, 1969; Jones and Powell, 1970; Van Hoesen et al., 1972; Chavis and Pandya, 1974, 1976; Van Hoesen and Pandya, 1975; Seltzer and Pandya, 1976; Barbas and Mesulam, 1981, 1985; Morel et al., 1993; Romanski et al., 1995).

In addition to anatomical similarities, the results of this current experiment indicate that the auditory behavioral impairments that follow damage to the ST cortex are similar in nature to the visual behavioral impairments that follow damage to the IT cortex. This is particularly so when one examines the effects of ST cortex and IT cortex lesions on auditory DMS and visual DMS behavior, respectively. In both cases, the lesions generally have little effect on the rate of forgetting; i.e., the rate at which information is forgotten across the delay periods changes very little from preoperative levels. Rather, ST cortex and IT cortex lesions generally tend to depress performance across all of the delay periods, including the shortest, indicating that both types of lesions result in more than simply an inability to remember auditory and visual information, respectively. Together, the anatomical and behavioral data support the suggestion raised by Mishkin (1982) that the ST cortex plays a role in auditory information processing similar to that played by the IT cortex for visual information processing.

One consequence of the idea that the neural basis of information processing in the visual and auditory systems may be similar is that it may offer a certain degree of predictive power. For example, the visual system seems to be arranged hierarchically into two cortical pathways, a ventral stream that projects to IT cortex that is primarily involved in object recognition, and a dorsal stream that projects to parietal cortex that is primarily responsible for spatial and visuomotor functions (Mishkin et al., 1983; Ungerleider, 1986; Eacott and Gaffan, 1991) From a behavioral stand-

TEO resulting in greater perceptual deficits and damage to area TE resulting in greater memory deficits (Iwai and Mishkin, 1968, 1969; Cowey and Gross, 1970; Iwai, 1985). Thus one might argue that IT lesions impair equivalence behavior in monkeys because such lesions, which include area TEO, impaired perceptual processes. Perhaps if the damage had been confined to area TE, then performance on tests of equivalence behavior would have been normal. In fact, damage to area TE also results in visual perceptual deficits such as impairments in global stereopsis (Cowey and Porter, 1979), object transforms (Weiskrantz and Saunders, 1984), and transfer from a three-dimensional object to a two-dimensional representation of the object (Holmes and Gross, 1984). Thus even animals with area TE damage probably do not perceive the world in exactly the same manner as normal animals do. 
point, monkeys with lesions of the ventral stream are impaired on object- but not spatial-discrimination tasks, with the reverse deficit holding true for monkeys with lesions to the dorsal stream (Mishkin et al., 1983).

A similar organization might be present in the auditory system. For example, primary auditory cortex (Kam and Kalt) projects to the surrounding parakoniocortex, in particular area PaAlt (Pandya et al., 1969; Pandya and Kuypers, 1969; Pandya and Vignolo, 1969; Jones and Powell, 1970; Morel et al., 1993). From this point, one can envision two pathways, a "ventral" stream consisting of projections to areas Ts3, Ts2, and Ts1 that is responsible for the recognition of sounds, and a "dorsal" system consisting of projections to Tpt and then area PG of the parietal lobe that is responsible for the processing of information related to the spatial localization of a sound source. In fact, it has been noted that Tpt seems architectonically more similar to PG than to Ts3, its neighboring region on the ST cortex (Sanides, 1972; Pandya and Sanides, 1973), and in addition, area Tpt projects to the parahippocampal cortex (Tranel et al., 1988), which is thought to be important for spatial information processing (Suzuki, 1994; Suzuki and Amaral, 1994). Furthermore, electrophysiological recordings of area Tpt in monkeys (Leinonen et al., 1980) have shown that the activity of cells in this region are significantly influenced by the location of the sound source with respect to the monkey's head. Thus, Tpt might be one station in a dorsal auditory pathway responsible for the spatial localization of a sound source.

Behaviorally, there is some evidence in favor of two auditory streams. For example, just as lesions to the dorsal visual stream have no effect on visual-pattern discrimination, lesions to Tpt and area PG, which constituted the hypothesized analogous dorsal stream in the auditory modality, do not affect the ability of monkeys to discriminate species-specific calls (Heffner and Heffner, 1984, 1986a). On the other hand, discrimination of speciesspecific sounds is impaired after lesions to the entire ST cortex, which houses all of the components of the proposed ventral system. It will be experiments of this sort and those such as the recent electrophysiological examinations of ST cortex (Rauschecker et al., 1995) that will ultimately determine the similarities or differences in how information is processed in the auditory and visual cortical areas.

\section{REFERENCES}

Barbas H, Mesulam M-M (1981) Organization of afferent input to subdivisions of area 8 in the rhesus monkey. J Comp Neurol 200:407-431.

Barbas H, Mesulam M-M (1985) Cortical afferent input to the principalis region of the rhesus monkey. Neuroscience 15:619-637.

Bender DB (1973) Visual sensitivity following inferotemporal and foveal prestriate lesions in the rhesus monkey. J Comp Physiol Psychol 84:613-621.

Bender DB, Gross CG (1981) Backward masking in monkeys after foveal prestriate and inferior temporal cortex lesions. Physiol Psychol 9:257-259.

Boussaoud D, Desimone R, Ungerleider LG (1991) Visual topography of area TEO in the macaque. J Comp Neurol 306:554-575.

Butter CM (1968) The effect of discrimination training on pattern equivalence in monkeys with inferotemporal and lateral striate lesions. Neuropsychologia 6:27-40.

Butter CM, Doehrman SR (1968) Size discrimination and transposition in monkeys with striate and temporal lesions. Cortex 4:35-46.

Butter CM, Gekoski WL (1966) Alterations in pattern equivalence following inferotemporal and lateral striate lesions in rhesus monkeys. J Comp Physiol Psychol 61:309-312.

Butter CM, Hirtzel M (1970) Impairment in sampling visual stimuli in monkeys with inferotemporal lesions. Physiol Behav 5:369-370.
Butter CM, Mishkin M, Rosvold HE (1965) Stimulus generalization in monkeys with inferotemporal and lateral occipital lesions. In: Stimulus generalization (Mostofsky DI, ed), pp 119-133. Stanford, CA: Stanford UP.

Chavis D, Pandya DN (1974) Frontal lobe projections of the cortical sensory association areas of the rhesus monkey. Trans Am Neurol Assoc 99:192-195.

Chavis DA, Pandya DN (1976) Further observations on corticofrontal connections in the rhesus monkey. Brain Res 117:369-386.

Chow KL, Survis J (1958) Retention of overlearned visual habit after temporal cortical ablation in monkey. Arch Neurol Psychiatry 79:640-646.

Colombo M, D'Amato MR (1986) A comparison of visual and auditory short-term memory in monkeys (Cebus apella). Q J Exp Psychol [B] 38:425-448.

Colombo M, Graziano M (1994) Effects of auditory and visual interference on auditory-visual delayed matching to sample in monkeys ( $\mathrm{Ma}$ caca fascicularis). Behav Neurosci 108:636-639.

Colombo M, D'Amato MR, Rodman HR, Gross CG (1990) Auditory association cortex lesions impair auditory short-term memory in monkeys. Science 247:336-338.

Colombo M, Eickhoff AE, Gross CG (1993) The effects of inferior temporal and dorsolateral frontal lesions on serial-order behavior and visual imagery in monkeys. Cog Brain Res 1:211-217.

Cordeau JP, Mahut H (1964) Some long-term effects of temporal lobe resections on auditory and visual discrimination in monkeys. Brain 87:177-190.

Costalupes JA (1984) Superior temporal lesions and delayed auditory matching in monkeys. Exp Neurol 84:478-483.

Cowey A, Dewson JH (1972) Effects of unilateral ablation of superior temporal cortex on auditory sequence discrimination in Macaca тиlatta. Neuropsychologia 10:279-289.

Cowey A, Gross CG (1970) Effects of foveal prestriate and inferotemporal lesions on visual discrimination by rhesus monkeys. Exp Brain Res 11:128-144.

Cowey A, Porter J (1979) Brain damage and global stereopsis. Proc R Soc Lond [Biol] 204:399-407.

Cowey A, Weiskrantz L (1963) A perimetric study of visual field defects in monkeys. Q J Exp Psychol 15:91-115.

Cowey A, Weiskrantz L (1967) A comparison of the effects of inferotemporal and striate cortex lesions on the visual behavior of rhesus monkeys. Q J Exp Psychol 19:246-253.

Cowey A, Weiskrantz L (1976) Auditory sequence discrimination in $M a$ caca mulatta: the role of the superior temporal cortex. Neuropsychologia $14: 1-10$.

Craik FIM, Lockhart RS (1972) Levels of processing: a framework for memory research. J Verb Learn Verb Behav 11:671-684.

D'Amato MR (1973) Delayed matching and short-term memory in monkeys. In: The psychology of learning and motivation: advances in theory and research, Vol 7 (Bower GH, ed), pp 227-269. New York: Academic.

D'Amato MR, Colombo M (1985) Auditory matching-to-sample in monkeys (Cebus apella). Anim Learn Behav 13:375-382.

D'Amato MR, Colombo M (1988a) On tonal pattern perception in monkeys (Cebus apella). Anim Learn Behav 16:417-424.

D'Amato MR, Colombo M (1988b) Representation of serial order in monkeys (Cebus apella). J Exp Psychol Anim Behav Processes 14:131-139.

D'Amato MR, Salmon DP (1982) Tune discrimination in monkey (Cebus apella) and in rats. Anim Learn Behav 10:126-134.

D'Amato MR, Salmon DP (1984) Processing of complex auditory stimuli (tunes) by rats and monkeys (Cebus apella). Anim Learn Behav 12:184-194.

D'Amato MR, Salmon DP, Colombo M (1985) Extent and limits of the matching concept in monkeys (Cebus apella). J Exp Psychol Anim Behav Processes 11:35-51.

Dean P (1974) Choice reaction times for pattern discriminations in monkeys with inferotemporal lesions. Neuropsychologia 12:465-476.

Dean P (1976) Effects of inferotemporal lesions on the behavior of monkeys. Psychol Bull 83:41-71.

Dean P (1982) Visual behavior in monkeys with inferotemporal lesions. In: Analysis of visual behavior (Ingle DJ, Goodale MA, Mansfield RJW, eds), pp 587-628. Cambridge, MA: MIT.

Desimone R, Gross CG (1979) Visual areas in the temporal cortex of the macaque. Brain Res 178:363-380. 
Dewson III JH (1977) Preliminary evidence of hemispheric asymmetry of auditory function in monkeys. In: Lateralization in the nervous system (Harnard S, Doty RW, Goldstein L, Jaynes J, Krauthamer G, eds), pp 63-71). New York: Academic.

Dewson III JH (1978) Some behavioral effects of removal of superior temporal cortex in the monkey. In: Recent advances in primatology, Vol 1: Behavior (Chivers DJ, Herbert J, eds), pp 763-768. London: Academic.

Dewson III JH (1979) Toward an animal model of auditory cognitive function. In: The neurological basis of language disorders in children: methods and directions for research (Ludlow CL, Doran-Quine ME, eds), pp 19-28. Washington, DC: GPO.

Dewson III JH, Cowey A, Weiskrantz L (1970) Disruptions of auditory sequences by unilateral and bilateral cortical ablations of superior temporal gyrus in the monkey. Exp Neurol 28:529-548.

Dewson III JH, Pribram KH, Lynch JC (1969) Effects of ablations of temporal cortex upon speech sound discrimination in the monkey. Exp Neurol 24:579-591.

Eacott MJ, Gaffan D (1991) The role of monkey inferior parietal cortex in visual discrimination of identity and orientation of shapes. Behav Brain Res 46:95-98.

Eacott MJ, Gaffan D, Murray EA (1994) Preserved recognition memory for small sets, and impaired stimulus identification for large sets, following rhinal cortex ablations in monkeys. Eur J Neurosci 6:1466-1478.

Evarts EV (1952) Effect of auditory cortex ablation on frequency discrimination in monkey. J Neurophysiol 15:443-448.

Felleman DJ, Van Essen DC (1991) Distributed hierarchical processing in the primate cerebral cortex. Cereb Cortex 1:1-47.

Fuster JM, Bauer RH, Jervey JP (1981) Effects of cooling inferotemporal cortex on performance of visual memory tasks. Exp Neurol 71:398-409.

Gaffan D (1977) Response coding in recall of colours by monkeys. Q J Exp Psychol 29:597-605.

Gaffan D, Harrison S (1991) Auditory-visual associations, hemispheric specialization and temporal-frontal interaction in the rhesus monkey. Brain 114:2133-2144.

Gross CG (1973) Inferotemporal cortex and vision. In: Progress in physiological psychology, Vol 5 (Stellar E, Sprague JM, eds), pp 77-123. New York: Academic.

Gross CG, Rodman HR (1992) Inferior temporal cortex: neuronal properties and connections in adult and infant macaques. In: The visual system from genesis to maturity (Lent R, ed), pp 245-266. Boston: Birkhauser.

Gross CG, Bender DB, Rocha-Miranda CE (1969) Visual receptive fields of neurons in inferotemporal cortex of the monkey. Science 166:1303-1306.

Gross CG, Rodman HR, Gochin PM, Colombo MW (1993) Inferior temporal cortex as a pattern recognition device. In: Computational learning and cognition (Baum EB, ed), pp 44-73. Philadelphia: Siam.

Hauser MD, Andersson K (1994) Left hemispheric dominance for processing vocalizations in adult, but not infant, rhesus monkeys: field experiments. Proc Natl Acad Sci 91:3946-3948.

Heffner HE, Heffner RS (1984) Temporal lobe lesions and perception of species-specific vocalizations by macaques. Science 226:75-76.

Heffner HE, Heffner RS (1986a) Effect of unilateral and bilateral auditory cortex lesions on the discrimination of vocalizations by Japanese macaques. J Neurophysiol 56:683-701.

Heffner HE, Heffner RS (1986b) Hearing loss in Japanese macaques following bilateral auditory cortex lesions. J Neurophysiol 55:256-271.

Heffner HE, Heffner RS (1989) Effect of restricted cortical lesions on absolute thresholds and aphasia-like deficits in Japanese macaques. Behav Neurosci 103:158-169.

Heffner HE, Heffner RS (1990) Effect of bilateral auditory cortex lesions on absolute thresholds in Japanese macaques. $J$ Neurophysiol 64:191-205.

Herman LM (1980) Cognitive characteristics of dolphins. In: Cetacean behavior: mechanisms and function (Herman LM, ed), pp 363-429. New York: Wiley.

Herman LM, Forestell PH (1985) Short-term memory in pigeons: modality-specific or code-specific effects? Anim Learn Behav 13:463-465.

Heywood CA, Gaffan D, Cowey A (1995) Cerebral achromatopsia in monkeys. Eur J Neurosci 7:1064-1073

Holmes EJ, Gross CG (1984) Stimulus equivalence after inferior temporal lesions in monkeys. Behav Neurosci 98:898-901.
Honig WK (1978) Studies of working memory in the pigeon. In: Cognitive processes in animal behavior (Hulse SE, Fowler H, Honig WK, eds), pp 211-248. Hillsdale, NJ: Lawrence Erlbaum Associates.

Honig WK, Thompson RKR (1982) Retrospective and prospective processing in animal working memory. In: The psychology of learning and motivation: advances in research and theory, Vol 16 (Bower GH, ed), pp 239-283). New York: Academic.

Horel JA, Pytko DE (1982) Behavioral effect of local cooling in temporal lobe of monkeys. J Neurophysiol 47:11-22.

Horel JA, Voytko ML, Salsbury KG (1984) Visual learning suppressed by cooling the temporal pole. Behav Neurosci 98:310-324.

Hull CL (1943) Principles of behavior. New York: Appleton-CenturyCrofts.

Hunter WS (1913) The delayed reaction in animals and children. Behavior Monographs 2, Serial Number 6.

Ibuka N, Kubota K, Iwai E (1974) Ablation of a small circumscribed portion of the inferotemporal cortex and a delayed matching-to-sample task. In: Contemporary primatology (Ibuka N, ed), pp 224-229. Basel, Switzerland: Karger.

Iversen SD, Mishkin M (1973) Comparison of superior temporal and inferior prefrontal lesions on auditory and non-auditory tasks in rhesus monkeys. Brain Res 55:355-367.

Iwai E (1985) Neuropsychological basis of pattern vision in macaque monkeys. Vision Res 25:425-439.

Iwai E, Mishkin M (1968) Two visual foci in the temporal lobe of monkeys. In: Neuropsychological basis of learning and behavior (Yoshii N, Buchwald NA, eds), pp 23-33. Osaka, Japan: Osaka UP.

Iwai E, Mishkin M (1969) Further evidence of the locus of the visual area in the temporal lobe of the monkey. Exp Neurol 25:585-594.

Jerison HJ, Neff WD (1953) Effect of cortical ablation in the monkey on discrimination of auditory patterns. Fed Proc 12:73-74.

Jones EG, Powell TPS (1970) An anatomical study of converging sensory pathways within the cerebral cortex of the monkey. Brain 93:793-820.

Kojima S (1980) Short-term memory in the rhesus monkey: a behavioral analysis of delayed-response performance. J Exp Anal Behav 33:359-368

Konorski J (1959) A new method of physiological investigation of recent memory in animals. Bull Acad Polonaise Sci [Biol] 7:115-117.

Kovner R, Stamm JS (1972) Disruption of short-term visual memory by electrical stimulation of inferotemporal cortex in the monkey. J Comp Physiol Psychol 81:163-172.

Leinonen L, Hyvarinen J, Sovijarvi ARA (1980) Functional properties of neurons in the temporo-parietal association cortex of awake monkey. Exp Brain Res 39:203-215.

Massopust Jr LC, Wolin LR, Frost V (1970) Increases in auditory middle frequency discrimination thresholds after cortical ablations. Exp Neurol 28:299-307.

Massopust Jr LC, Wolin LR, Meder R, Frost V (1967) Changes in auditory frequency discrimination thresholds after temporal cortex ablations. Exp Neurol 19:245-255.

Mishkin M (1982) A memory system in the monkey. Philos Trans R Soc Lond [Biol] 298:85-92.

Mishkin M, Ungerleider LG, Macko KA (1983) Object vision and spatial vision: two cortical pathways. Trends Neurosci 6:414-417.

Morel A, Garraghty PE, Kaas JH (1993) Tonotopic organization, architectonic fields, and connections of auditory cortex in macaque monkeys. J Comp Neurol 335:437-459.

Neff WD (1961a) Discriminatory capacity of different divisions of the auditory system. In: Brain and behavior, Vol 1 (Brazier MAB, ed), pp 205-262. Washington, DC: AIBS.

Neff WD (1961b) Neural mechanisms of auditory discrimination. In: Sensory communication (Rosenblith WA, ed), pp 259-278. New York: Wiley.

Orbach J, Fantz RL (1958) Differential effects of temporal neocortical resections on overtrained and nonovertrained visual habits in monkeys. J Comp Physiol Psychol 51:126-129.

Pandya DN, Kuypers HGJM (1969) Cortico-cortical connections in the rhesus monkey. Brain Res 13:13-36.

Pandya DN, Sanides F (1973) Architectonic parcellation of the temporal operculum in rhesus monkey and its projection pattern. Z Anat Entwicklungsgesch 139:127-161.

Pandya DN, Vignolo LA (1969) Interhemispheric projections of the parietal lobe in the rhesus monkey. Brain Res 15:49-65. 
Pandya DN, Yeterian EH (1985) Architecture and connections of cortical association areas. In: Cerebral cortex, Vol 4, Association and auditory cortices (Peters A, Jones EG, eds), pp 3-61. New York: Plenum.

Pandya DN, Hallett M, Mukherjee SK (1969) Intra- and interhemispheric connections of the neocortical auditory system in the rhesus monkey. Brain Res 14:49-65.

Pandya DN, Seltzer B, Barbas H (1988) Input-output organization of the primate cerebral cortex. In: Comparative primate biology, Vol 4, Neurosciences (Steklis HD, Erwin J, eds), pp 39-80. New York: Alan Liss.

Peretz I, Kolinsky R, Tramo M, Labrecque R, Hublet C, Demeurisse G, Belleville S (1994) Functional dissociations following bilateral lesions of auditory cortex. Brain 117:1283-1301.

Petersen M, Beecher M, Zoloth S, Moody D, Stebbins W (1978) Neural lateralization of species-specific vocalizations by Japanese macaques (Macaca fuscata). Science 202:324-327.

Pratt SR, Iversen SD (1978) Selective cortical lesions and auditory behavior in the monkey. In: Recent advances in primatology, Vol 1, Behavior (Chivers DJ, Herbert J, eds), pp 807-809. London: Academic.

Rauschecker JP, Tian B, Hauser M (1995) Processing of complex sounds in the macaque nonprimary auditory cortex. Science 268:111-114.

Ravizza RJ, Belmore SM (1978) Auditory forebrain: evidence from anatomical and behavioral experiments involving human and animal subjects. In: Handbook of behavioral neurobiology, Vol 1, Sensory integration (Masterson RB, ed), pp 459-501. New York: Plenum.

Roberts WA, Grant DS (1976) Studies of short-term memory in the pigeon using the delayed matching-to-sample procedure. In: Processes of animal memory (Medin DL, Roberts WA, Davis RT, eds), pp 79-112. Hillsdale, NJ: Erlbaum.

Rodman HR (1994) Development of inferior temporal cortex in the monkey. Cereb Cortex 5:484-498.

Roitblat HL (1980) Codes and coding processes in pigeon short-term memory. Anim Learn Behav 8:341-351.

Romanski LM, Bates JF, Goldman-Rakic PS (1995) Selective connections of the superior temporal auditory region with the prefrontal cortex in the macaque monkey. Soc Neurosci Abstr 21:932.

Sahgal A, Iversen SD (1978a) Categorization and retrieval after selective inferotemporal lesions in monkeys. Brain Res 146:341-350.

Sahgal A, Iversen SD (1978b) The effects of foveal prestriate and inferotemporal lesions on matching to sample behavior in monkeys. Neuropsychologia 16:391-406.

Samson S, Zatorre RJ (1992) Learning and retention of melodic and verbal information after unilateral temporal lobectomy. Neuropsychologia 30:815-826.

Sanides F (1972) Representation in the cerebral cortex and its areal lamination patterns. In: The structure and function of nervous tissue, Vol V, Structure III and physiology III (Bourne GH, ed), pp 329-453. New York: Academic.

Seltzer B, Pandya DN (1976) Some cortical projections to the parahippocampal area in the rhesus monkey. Exp Neurol 50:146-160.

Stepien LS, Cordeau JP, Rasmussen T (1960) The effects of temporal lobe and hippocampal lesions on auditory and visual recent memory in monkeys. Brain 83:470-489.

Sutherland NS, Mackintosh NJ (1971) Mechanisms of animal discrimination learning. New York: Academic.

Suzuki WA (1994) What can neuroanatomy tell us about the functional components of the hippocampal memory system? Behav Brain Sci 17:496-498.
Suzuki WA, Amaral DG (1994) Perirhinal and parahippocampal cortices of the macaque monkey: cortical afferents. J Comp Neurol 350:497-533.

Symmes D (1965) Flicker discrimination by brain-damaged monkeys. J Comp Physiol Psychol 60:470-473.

Tanaka K, Saito H-A, Fukada Y, Moriya M (1991) Coding visual images of objects in the inferotemporal cortex of the macaque monkey. J Neurophysiol 66:170-189.

Tranel D, Brady DR, Van Hoesen GW, Damasio AR (1988) Parahippocampal projections to posterior auditory association cortex (area $\mathrm{Tpt}$ ) in old-world monkeys. Exp Brain Res 70:406-416.

Ungerleider LG (1986) The corticocortical pathways for object recognition and spatial perception. Exp Brain Res 11:21-37.

Van Hoesen GW, Pandya DN (1975) Some connections of the entorhinal (area 28) and perirhinal (area 35) cortices of the rhesus monkey. I. Temporal lobe afferents. Brain Res 95:1-24.

Van Hoesen GW, Pandya DN, Butters N (1972) Cortical afferents to the entorhinal cortex of the rhesus monkey. Science 175:1471-1473.

von Bonin G (1938) The cerebral cortex of the cebus monkey. J Comp Neurol 69:181-227.

von Bonin G, Bailey P (1947) The neocortex of Macaca mulatta. Urbana, IL: University of Illinois.

Wagner AR (1981) SOP: a model of automatic memory processing in animal behavior. In: Information processing in animals: memory mechanisms (Spear NE, Miller RR, eds), pp 5-47. Hillsdale, NJ: Lawrence Erlbaum Associates.

Wallace J, Steinert PA, Scobie SR, Spear NE (1980) Stimulus modality and short-term memory in rats. Anim Learn Behav 8:10-16.

Webster MJ, Bachevalier J, Ungerleider LG (1994) Connections of inferior temporal areas TEO and TE with parietal and frontal cortex in macaque monkeys. Cereb Cortex 5:470-483.

Webster MJ, Bachevalier J, Ungerleider LG (1995) Transient subcortical connections of inferior temporal areas TE and TEO in infant macaque monkeys. J Comp Neurol 352:213-226.

Webster MJ, Ungerleider LG, Bachevalier J (1991) Connections of inferior temporal areas TE and TEO with medial temporal-lobe structures in infant and adult monkeys. J Neurosci 11:1095-1116.

Wegener JG (1968) The effect of cortical lesions on auditory and visual discrimination behavior in monkeys. Cortex 4:203-232.

Wegener JG (1976) Auditory and visual discrimination following lesions of the anterior supratemporal plane in monkeys. Neuropsychologia 14:161-173.

Weiskrantz L, Cowey A (1963) Striate cortex lesions and visual acuity of the rhesus monkey. J Comp Physiol Psychol 56:225-231.

Weiskrantz L, Mishkin M (1958) Effects of temporal and frontal cortical lesions on auditory discrimination in monkeys. Brain 81:406-414.

Weiskrantz L, Saunders RC (1984) Impairments of visual object transforms in monkeys. Brain 107:1033-1072.

Wilson M (1968) Inferotemporal cortex and the processing of visual information in monkeys. Neuropsychologia 6:135-140.

Zatorre RJ (1985) Discrimination and recognition of tonal melodies after unilateral cerebral excisions. Neuropsychologia 23:31-41.

Zatorre RJ, Evans AC, Meyer E (1994) Neural mechanisms underlying melodic perception and memory for pitch. J Neurosci 14:1908-1919. 